\title{
DNA replication dynamics during erythrocytic schizogony in the malaria parasites Plasmodium falciparum and Plasmodium knowlesi
}

\author{
Jennifer McDonald ${ }^{1}$, Catherine J. Merrick ${ }^{1 \#}$ \\ ${ }^{1}$ Department of Pathology, University of Cambridge, Tennis Court Road, Cambridge, CB2 1QP, UK \\ \# Address correspondence to: \\ Catherine J. Merrick, Department of Pathology, Cambridge University, Tennis Court Road, Cambridge, CB2 \\ 1QP, UK \\ Tel: (+44) 1223333330 \\ Email: cjm48@cam.ac.uk
}

\section{ABSTRACT}

Malaria parasites are unusual, early-diverging protozoans with non-canonical cell cycles. They do not undergo binary fission, but divide primarily by schizogony. This is a syncytial mode of replication involving asynchronous production of multiple nuclei within the same cytoplasm, culminating in a single mass cytokinesis event. The rate and efficiency of parasite replication is fundamentally important to malarial disease, which tends to be severe in hosts with high parasite loads. Here, we have studied for the first time the dynamics of schizogony in two human malaria parasite species, Plasmodium falciparum and Plasmodium knowlesi. These differ in their cell-cycle length, the number of progeny produced and the genome composition, among other factors. Comparing them could therefore yield new information about the parameters and limitations of schizogony. We report that the dynamics of schizogony differ significantly between these two species, most strikingly in the gap phases between successive nuclear replications, which are longer in $P$. falciparum and shorter, but more heterogenous, in $P$. knowlesi. In both species, gaps become longer as schizogony progresses, whereas each period of active replication grows shorter. In both species there is also extreme variability between individual cells, with some schizonts producing many more nuclei than others, and some individual nuclei arresting their replication for many hours while adjacent nuclei continue to replicate. The efficiency of schizogony is probably influenced by a complex set of factors in both the parasite and its host cell.

\section{INTRODUCTION}

The malaria parasite Plasmodium lies in an early-diverging protozoan lineage with many unusual features. One such feature is the type of cell cycle that this parasite pursues [1]. Plasmodium does not divide by binary fission - the well-studied process that occurs in almost all model systems from yeasts to human cells. Instead, division is primarily by schizogony, a unique syncytial process involving asynchronous replication of multiple nuclei within the same cytoplasm, prior to a mass cytokinesis event producing many - and not necessarily $2^{n}$ - daughter cells called merozoites [2].

Schizogony occurs first in hepatocytes and then repeatedly in erythrocytes, causing all the pathology of malaria. The process is clinically important because the rate of growth in erythrocytes determines parasitaemia, which often correlates with disease severity $[3,4]$. Parasitaemia in human malaria can vary greatly, partly because the human-infective Plasmodium species ( $P$. falciparum, $P$. vivax, P. malariae, $P$. vivax, P. knowlesi and $P$. ovale wallikeri \& curtsii) differ in their preferences for 
human erythrocytes. For example, $P$. vivax is restricted to invading scarce reticulocytes, $P$. malariae primarily invades older erythrocytes, and $P$. falciparum invades cells of all ages and can therefore reach high parasitaemias as extreme as $40 \%$ [5].

There are also other inter-species differences in the process of schizogony. Its duration varies from $\sim 72$ hours in $P$. malariae to $\sim 28$ hours in $P$. knowlesi and the number of merozoites produced per schizont varies from as many as $\sim 30$ in P. falciparum to $\sim 15$ in $P$. knowlesi. Exactly what limits merozoite numbers to a characteristic range for each species is unknown, but it is evidently not the fundamental size of the human erythrocyte, nor the duration of schizogony. Moving from the cell-biological to the molecular level, the composition of Plasmodium genomes also varies considerably between species. $P$. falciparum has an extremely biased genome at $~ 81 \% A / T$ [6] whereas $P$. vivax and $P$. knowlesi have $A / T$ contents of only 60 and $61 \%$. The reasons for these differences, and their potential influence upon the speed and fidelity of DNA replication, are unknown.

To better understand the dynamics of schizogony, we have compared the process in the only two species that are amenable to culture, $P$. falciparum and $P$. knowlesi. These differ in parameters such as genome content, cell cycle period and merozoite number. Little has been published on $P$. knowlesi replication but we have previously explored replication dynamics in $P$. falciparum [7]. To do this we created a parasite line that allows de novo DNA replication to be followed at high resolution via the incorporation of pulse-labels of modified nucleotides [8]. Thus, we defined replication dynamics at the single-molecule level [7] and, together with prior work at the cellular level $[2,9]$, this provided an overall picture of $P$. falciparum erythrocytic schizogony. Here, we have added considerable detail to that picture and used the same replication-labelling technology to compare $P$. knowlesi - a species that completes schizogony twice as fast, makes fewer merozoites, and has a more balanced genome composition.

To summarise what is already known about $P$. falciparum schizogony, the first round of DNA replication begins more than halfway through the cell cycle (which is theoretically $48 \mathrm{~h}$, actually $\sim 42-$ $50 \mathrm{~h}$ in different strains and culture systems). Four or five rounds of nuclear replication occur and there is karyokinesis at each round [9], without cytokinesis or conventional G1/G2 phases [2, 10, 11]. Duplication of the centriolar plaque (the Plasmodium centriole-equivalent) has been proposed to initiate each replication. Asynchrony, which is clearly seen in the early rounds, has been linked to partitioning of centriolar plaques, with the nucleus that receives the more mature plaque commencing its next replication first [2]. Without the benefit of DNA replication labelling, prior work could not define the later replicative rounds precisely, as nuclei become very crowded inside the erythrocyte, but the final round of replication was suggested to be synchronous $[12,13]$. The degree of synchrony has since been disputed, most recently via high-resolution electron-microscopy data $[2,14]$. Indeed, it is unclear how a final synchronous round would be coordinated if centriolar plaques of differing maturity do drive the rate of replication. Other unanswered questions include: is there a limit on the number of nuclei that can replicate at once, perhaps via a limiting factor, and how is this overcome if the final round is synchronous? Do all successive rounds of nuclear replication take the same time? Are there consistent gaps between replicative rounds (G1 or G2 equivalents), or do some nuclei 'stall' - adding to asynchrony and suggesting a DNA replication checkpoint?

At the single-molecule level, our recent work defined the average speed of replication forks and spacing of replication origins in $P$. falciparum schizogony at $1.2 \mathrm{~kb} / \mathrm{min}$ and $65 \mathrm{~kb}$ between origins - broadly similar to parameters in other eukaryotic cells. These averages changed by $\sim 30 \%$ over the 
course of schizogony, with the fastest fork movement and most widely-spaced origins occurring early on. The opposite pattern occurs in human cells, where replication speed is usually limited by cellular nucleotide pools and becomes fastest towards the end of S-phase as nucleotide production peaks. By contrast, in P. falciparum, we speculated that the nucleotide pool could become increasingly limiting as more nuclei replicate at once, and/or that DNA condensation or accumulated DNA damage might limit the pace of replication [7]. In P. knowlesi, fewer nuclei are packed into each cell and the genome composition is different from that of $P$. falciparum, so the parameters of replication could conceivably be very different. Accordingly, we have now measured replication parameters at both the cellular and single-molecule levels in both species.

\section{RESULTS}

\section{$\underline{\text { Timecourse experiments across S-phase in P. falciparum and P. knowlesi }}$}

To follow DNA replication dynamics throughout schizogony in both $P$. falciparum and $P$. knowlesi, we first transfected $P$. knowlesi with a thymidine-kinase-expressing plasmid, as previously performed in P. falciparum [8]. We confirmed that this permitted the labelling of DNA replication with modified nucleotides (bromo/chloro/iodo/ethyl-deoxyuridine (BrdU/CldU/IdU/EdU)), and that parasite behaviour in terms of fitness and cell-cycle timing was not overtly disturbed.

We then conducted time-course experiments with highly synchronised cultures of both lines. Synchronisation was achieved by purifying late-stage schizonts, allowing $1 \mathrm{~h}$ of reinvasion, then purifying the resultant ring-stages to yield a $1 \mathrm{~h}$ window around the point of invasion (termed ' $0 \mathrm{hpi}$ '). Aliquots of parasites were pulse-labelled with EdU for 30 minutes every hour throughout the period of DNA replication, then immediately fixed and prepared for microscopy. All the nuclei in each cell were detected with a DNA stain, and all the actively replicating nuclei were detected via EdU click chemistry. Centrin foci were also detected by immunofluorescence [2], to quantify the centriolar plaques associated with each nucleus. Figure $1 \mathrm{~A}$ shows a schematic of the experimental setup. In both species, additional experiments were then conducted, using two distinguishable pulses of modified nucleotides at increasing time intervals (Figure 1B,C). This allowed the detection not only of nuclei actively replicating at time $x$, but also of nuclei that had been replicating at time $x-y$. Thus, we could determine the length of each replicative round and of the gaps between rounds.

\section{The length of S-phase is proportional to the overall length of erythrocytic schizogony}

To measure the overall parameters of S-phase, we first counted all the nuclei, and all the replicating nuclei, in 100 cells at each timepoint listed in Figure $1 \mathrm{~A}$ (Supp Figure 1). P. falciparum schizonts produced 5-28 nuclei while $P$. knowlesi produced 3-16 (Figure 2A, B). Median numbers of nuclei at the most mature timepoint were 11 for $P$. falciparum and 6 for $P$. knowlesi, but the numbers produced in both species were highly variable. Amongst populations of late-stage schizonts, rare cells were still visible with only 4 or 2 nuclei respectively (Figure 2A, B). This could be because a small minority of cells were very delayed in starting S-phase, or because a minority underwent fewer/slower rounds of replication. Favouring the latter explanation, the onset of S-phase for the majority of cells was quite synchronous (Figure 2C, D), particularly in P. knowlesi, where $~ 60 \%$ of cells commenced replication within a single hour at 22-23hpi.

The overall length of S-phase was estimated as the time between the first appearance of cells with actively replicating DNA and the time when nuclear numbers stopped increasing. In $P$. 
falciparum, cells with replicating DNA began to appear at 31-32hpi and cells with more than 1 nucleus (having completed the first S-phase) began to appear at $32 \mathrm{hpi}$ (Figure 2A, C). Numbers of nuclei stopped increasing at 46-47hpi, making 15 hours the total S-phase period across the population. In P. knowlesi, replication started at $22 \mathrm{hpi}, 2 \mathrm{n}$ cells began to appear at $23 \mathrm{hpi}$, and numbers of nuclei stopped increasing at 31-32hpi (coinciding with a sharp drop in EdU labelling from $31 \mathrm{hpi}$ ), i.e. an S-phase period of only $9 \mathrm{~h}$ (Figure 2B, D). Notably, this P. knowlesi timecourse was followed until almost all the schizonts had burst at $33 \mathrm{hpi}$, confirming a lag of just $2 \mathrm{~h}$ between the cessation of DNA replication and the completion of reinvasion. In $P$. falciparum, this first timecourse was not followed through to the end of reinvasion but a subsequent EdU/BrdU labelled timecourse would confirm that $P$. falciparum showed substantial reinvasion by $48 \mathrm{hpi}$ (Supp Figure 2), and so has a similar window of $\sim 2 \mathrm{~h}$ between the end of S-phase and reinvasion. Within this window, daughter merozoites must be fully assembled and cytokinesis by budding must occur.

These data showed that the proportion of the cell cycle occupied by S-phase was very similar in both species, at $30 \%$ ( 15 of $48 \mathrm{~h}$, or 9 of $31 \mathrm{~h}$ ) and that the lag period between replication and reinvasion was similarly short in both species.

\section{There is no restriction on the number of nuclei that can replicate at once}

DNA replication in schizogony is clearly a stochastic, asynchronous process and different nuclei within the same cell can simultaneously experience either DNA replication or resting 'gap' phases [2, $7,9]$. We therefore examined whether there is a limit on the number of nuclei that can replicate simultaneously. If, for example, the pool of nucleotides or replication proteins is limiting, we should observe a limit on the number of nuclei per cell that can replicate their DNA within a 30-minute period. In fact, throughout schizogony this was not true. In the earliest stages of S-phase, the percentage of labelled nuclei was either 50 or $100 \%$, since cells had only 1 or 2 nuclei. Subsequently, however, cells appeared with anything from $20 \%$ to $100 \%$ of their nuclei replicating. The median percentage at most timepoints was $60-70 \%$ in $P$. falciparum and $50-60 \%$ in P. knowlesi (Figure 2E, F).

To obtain a clearer view of whether the number of nuclei replicating at once depended on the number of nuclei existing in a schizont, we re-plotted the data according to number of existing nuclei rather than hpi (Figure 2G, H). This confirmed the conclusion that a schizont with any number of nuclei could replicate any proportion of those nuclei within a 30-minute window. Notably, in $P$. knowlesi replication was rarely observed after the 8-9 nuclei stage (Figure $2 \mathrm{H}$ ), consistent with this species reaching a maximum of 16 nuclei, and with the final round of replication being quite, but not entirely, synchronous. By contrast, numbers of nuclei in $P$. falciparum schizonts were highly variable and cells with 15 or more nuclei were therefore binned together (Figure 2G), but there was no single number of nuclei (even above 15) at which replication ceased, indicating greater variability and asynchrony in $P$. falciparum.

\section{Patterns of DNA replication within nuclei vary from early to late schizogony}

In both $P$. falciparum and $P$. knowlesi, it was clear that the sub-nuclear distribution of activelyreplicating DNA during the 30-minute pulse-label varied in different nuclei (Figure 3A). The commonest pattern was a completely labelled nucleus with signal detected throughout the DAPIstained area, showing that DNA in all areas had replicated in the previous 30 minutes. Amongst these, and particularly notable in $P$. falciparum, was a subset of nuclei with homogenous but fainter staining, suggesting that DNA had replicated much less rapidly than in the common pattern: this 
appeared mostly in late-stage cells completing their presumptive final round of replication (Figure 3B, see P. falciparum at $44-45 \mathrm{hpi}, P$. knowlesi at $30 \mathrm{hpi}$, and quantification in Figure 3C, D). Other patterns included nuclei with only a partial area showing replication, and nuclei with discrete foci of replication: these appeared at low frequencies throughout both timecourses (Figure 3C).

To quantify these observations, we categorised replication patterns in the nuclei of 100 cells at each timepoint (Figure $3 \mathrm{C}$ ). We also quantified the amount of de novo DNA replication occurring within the pulse-label via the density of EdU staining per nucleus (Figure 3D). Marked differences appeared between $P$. falciparum and $P$. knowlesi. Firstly, nuclei with the 'partial' replication patterns made up $20-30 \%$ of the total in $P$. falciparum at all timepoints, but fewer than $10 \%$ of the total in $P$. knowlesi (Figure 3C). Secondly, the majority of nuclei in $P$. falciparum showed the faint staining pattern at $44-47 \mathrm{hpi}$, whereas in $P$. knowlesi this pattern increased slightly but never became dominant, appearing in only $\sim 20 \%$ of nuclei (Figure 3 C, D).

\section{Centriolar plaques are dynamic throughout schizogony}

Several previous studies have highlighted the importance of the centrosome equivalent - the centriolar plaque - in controlling nuclear replication $[2,15]$ (Figure 4A). We therefore quantified whether or not nuclei in each phase of replication were associated with detectable centriolar plaques, using foci of centrin as a marker. Centrin foci were not always detectable, consistent with the work of Simon et al. [15] (Figure 4B, Supp figure 1). Overall, $\sim 50 \%$ of nuclei had detectable centrin foci, varying from $<30 \%$ in the earliest and latest timepoints to $>60 \%$ at the midpoint of schizogony (37-42hpi in P. falciparum and $25-28 \mathrm{hpi}$ in P. knowlesi (Figure 4C)). Only a minority of nuclei, $\sim 25 \%$ at most timepoints, showed centrin foci as well as active DNA replication, while the majority showed either replication with no centrin, or centrin with no replication (Figure 4D). In both species there was some evidence that nuclei with centrin foci anti-correlated with actively replicating, centrin-negative nuclei (see for example 42hpi in Figure 4D). These observations point to a temporal separation between the start of DNA replication and the start of centriolar plaque elaboration and duplication.

The data were broken down into distinct types of centrin labelling: a single focus, two separate but adjacent foci, and two foci on opposite sides of the nucleus (Supp figure 1). These are thought to represent sequential events in preparation for karyokinesis (Figure 4A). Across 100 cells, in both $P$. falciparum and $P$. knowlesi, duplicated centrin foci were relatively rare, particularly in the opposite configuration, showing that this phase is very brief (Figure $4 \mathrm{C}$ ). About half of all nuclei with two foci showed DNA replication within the past 30 minutes, while the other half did not (Figure 4D). Finally, nuclei with centrin foci were never seen past the stage of 8-9 nuclei in $P$. knowlesi (Supp figure 3). This division was less sharp in $P$. falciparum, but the appearance of centrin foci did drop off in cells with more than 10 nuclei. In both species, it therefore appears than centriolar plaques are disassembled after the final round of DNA replication and karyokinesis. Beyond this, it was not possible to discern a clear relationship between the pattern of intranuclear DNA replication (full, partial, discrete foci, etc.) and the pattern of centrin foci (Supp figure 3).

\section{Replicative rounds become faster across the course of schizogony}

The foregoing experiments could not measure the time taken by each round of nuclear replication, because they provided only snapshots at successive timepoints. Therefore, we elaborated the 
protocol to use two successive, distinguishable pulse-labels (Figure 1B). Cultures were labelled with EdU for 30 minutes (as before), then with BrdU at 15-minute intervals up to 120 minutes in total. In a 1-n trophozoite, a single nucleus bearing both labels (Figure 5A) must have been replicating from at least the end of the first pulse to the start of the second, thus measuring the potential length of $S$ phase. Accordingly, when 30 minutes of EdU label was directly followed by 15 minutes of BrdU, Sphase could be $\geq 45$ minutes, but minimally $\sim 10$ minutes (requiring 5 active minutes in each pulse for replicating DNA to be detected with confidence (Supp figure 4)). Similarly, with a gap of 75 minutes separating the labels, S-phase must be at least 85 minutes. Figure $5 \mathrm{~B}$ quantifies this experiment for P. falciparum, showing that at least $70 \%$ of replication events in 1-n cells took $40-75$ minutes, but only $15 \%$ took 55-90 or 70-105, and almost none took $>85$ minutes (Figure 5B). (Notably, at the $45-$ minute interval, $35 \%$ of nuclei had completed replication within the first pulse but did not label at all with the second, i.e. they paused for at least an hour before karyokinesis. This percentage then dropped, suggesting that very few nuclei paused for $>75$ minutes before dividing and starting a second round.)

In multinucleate cells, the situation is more complex because a nucleus could potentially have completed replication during the first label, divided in the intervening period, and then picked up the second label in a subsequent replicative round. We assumed that if most replications took 40-75 minutes, this would be unlikely when the interval between labels was $\leq 30$ minutes, and therefore counted such double-labelled nuclei as being in the same replicative round. As ploidy increased there was a clear trend towards fewer nuclei being double-labelled at increasing time intervals (Figure 5B, compare 2 nuclei with $<6$ nuclei). Therefore, replicative rounds became progressively faster as ploidy increased.

In P. knowlesi (Figure 5C), the first replicative round took markedly longer, with $\sim 40 \%$ of nuclei taking 55-90 minutes, compared to only $15 \%$ of the equivalent nuclei in $P$. falciparum. In fact, $\sim 15 \%$ of nuclei took as long as $85-120$ minutes (Figure 5D). After this, however, subsequent replicative rounds seemed to speed up more than they did in $P$. falciparum. In $P$. knowlesi with $>6$ nuclei, only $\sim 25 \%$ of those nuclei replicated for $\geq 25$ minutes ( $\sim 25 \%$ of nuclei showed EdU+BrdU after the $15 \mathrm{~min}$ time interval), whereas in $P$. falciparum with $>6$ nuclei, $\sim 40 \%$ were still taking at least 25 minutes to complete a replicative round. In fact, in $P$. knowlesi schizonts with $>6$ nuclei, some nuclei probably entered the next round within 40 minutes (see Figure 5D, 30min interval).

\section{Gaps between replicative rounds become longer across the course of schizogony}

Finally, the interval-labelling protocol shown in Figures 1B was extended over longer periods, with intervals of up to $10 \mathrm{~h}$ (Figure $1 \mathrm{C}$ ). This was designed to assess the gaps between rounds of replication (and also to serve as a replicate, confirming the reproducibility of the initial datasets). Examples of the resultant labelling patterns are shown in Figure 6A. These were quantified in terms of the percentage of nuclei labelled with either one or both nucleotides across 50 cells (Figure 6B). (Of note, S-phase in $P$. falciparum proceeded similarly to the previous timecourse, occurring between $\sim 32-47$ hi, but S-phase in $P$. knowlesi was set forward by several hours in this experiment. In our hands, the cycle time of $P$. knowlesi is quite sensitive to different batches of human erythrocytes.)

The data indicated that a) gap phases are relatively long, b) they are longer in $P$. falciparum than $P$. knowlesi, and $c$ ) they become longer in both species over the course of S-phase. In $P$. falciparum, when sequential labels were separated by $2 \mathrm{~h}(41-43$ and $46-48 \mathrm{hpi})$ only $\sim 20 \%$ of nuclei were double-labelled, showing that relatively few nuclei commenced a second round within $2 \mathrm{~h}$ 
(Supp figure 5). With $3 \mathrm{~h}$ intervals, a greater proportion of nuclei re-entered S-phase: $\sim 50 \%$ of nuclei became double-labelled between $35-38 \mathrm{hpi}$, but this diminished to $\sim 40 \%$ and $20 \%$ at $38-41$ and 41 $44 \mathrm{hpi}$ (Figure 6B). Therefore, gaps increased over the course of S-phase (and/or progressively more nuclei did not re-enter replication at all), even as the time spent by each nucleus actively replicating became shorter as schizogony progressed (Figure 5). By contrast, in P. knowlesi, gap phases were generally shorter because markedly more nuclei became double-labelled within $2 \mathrm{~h}: ~ 50 \%$ of all nuclei in early stages, diminishing again to $35 \%$ and $20 \%$ at the final $2 \mathrm{~h}$ intervals (Figure $6 \mathrm{~B}$ ).

\section{$\underline{\text { Individual nuclei commonly arrest for long periods during schizogony }}$}

To address the question of heterogeneity in gaps - i.e. whether nuclei can arrest for long periods, or even permanently, while others in the same cell continue to replicate, we labelled cells for 30 minutes with EdU, followed by a continuous $5 \mathrm{~h} \mathrm{BrdU} \mathrm{label.} \mathrm{Nuclei} \mathrm{showing} \mathrm{EdU} \mathrm{but} \mathrm{no} \mathrm{BrdU}$ must have arrested for at least $5 \mathrm{~h}$ after a period of active replication: markedly longer than the common gap period of 2-3h suggested by Figure 6. Such nuclei did indeed appear often, and not exclusively in mature cells where many nuclei could have finished replicating already (Figure 7A). In relatively young $P$. falciparum cells with $\leq 6$ nuclei, $\sim 10 \%$ of all nuclei were arrested for at least $5 \mathrm{~h}$ (Figure $7 \mathrm{~B}$ ). In fact, this proportion fell to $\sim 5 \%$ in $>6$-nucleus schizonts, but the corresponding proportion of entirely unlabelled nuclei rose (i.e. those that had ceased to replicate even before the EdU pulse). In most individual cells, the number of arrested (EdU-only) nuclei was relatively low, 1-2 nuclei per cell, but rare cells were observed with more than half of their nuclei arrested for at least $5 \mathrm{~h}$ (Figure 7C).

This phenomenon was more prominent and occurred more at earlier times in $P$. knowlesi (Figure 7D-F). Even in relatively young schizonts, $\sim 15 \%$ of nuclei were unlabelled - i.e. they had ceased to replicate before the EdU pulse (see Figure 7E: 15\% of nuclei were arrested in cells with 26 nuclei, and similarly even in cells with 2-3 nuclei (Supp figure 6)). By contrast, far fewer nuclei were labelled with BrdU only, meaning that they were unlikely to have paused throughout the EdU pulse and then commenced replication again later on - which was a very common observation in $P$. falciparum. Instead, nuclei in $P$. knowlesi could either arrest long-term very early in schizogony, or go on to replicate multiple times with only short pauses between replicative rounds. This supports the observation from Figure 6 that the gaps between replicative rounds were shorter in $P$. knowlesi than P. falciparum.

Single-molecule replication dynamics do not differ significantly between $P$. falciparum and $P$. knowlesi

We previously measured the single-molecule dynamics of DNA replication in $P$. falciparum, determining that replication fork speed was $\sim 1.2 \mathrm{~kb} / \mathrm{min}$ and the spacing between adjacent replication origins was $\sim 65 \mathrm{~kb}$ [7]. Since the genome composition of $P$. knowlesi is very different, we sought to establish whether these parameters would differ in a less A/T-biased Plasmodium genome.

Our previous work employed the gold-standard DNA combing technique [16, 17], which linearises single DNA fibres on glass slides at a uniform stretching factor of $2 \mathrm{~kb} / \mu \mathrm{m}$. Cells are first pulse-labelled with two modified nucleosides, CldU and IdU, to generate DNA fibres in which the 
speed and directionality of replication forks can be measured (Figure 8A) [18]. Unfortunately this technique has recently become unavailable due to the discontinuation of the only antibody that reliably distinguishes CldU and IdU (previously used by all researchers in this field). The alternative double-label, EdU/BrdU, as used here in Figures 2-7, proved incompatible with DNA combing because EdU click chemistry gave poor results on the sialinized glass coverslips specifically required for DNA combing. Therefore, we reverted to the simpler technique of spreading DNA fibres from crude cell lysates on non-sialinized glass slides [19, 20]. This permitted EdU/BrdU labelling (Figure $8 B$ ), albeit at the expense of a uniform DNA stretching factor and the loss of very long fibres (DNA spreading tends to generate bundled fibres with only short lengths of single-stranded DNA, particularly when using parasite cells which have haemozoin debris in the cell lysate).

Figure $8 \mathrm{C}$ shows that replication fork speeds were not significantly different in the two species, although they trended faster in P. knowlesi. Replication origin spacing was identical in the two species (Figure 8D). Using the published DNA stretching factor of $2.59 \mathrm{~kb} / \mu \mathrm{m}$, calculated historically on DNA spreads from human cells [20], replication fork speeds could be calculated at 0.6$0.7 \mathrm{~kb} / \mathrm{min}$, and inter-origin spacing at $\sim 30 \mathrm{~kb}$, but these figures are lower than our earlier measurements on combed DNA fibres from $P$. falciparum [7], and are probably less accurate because the DNA spreading method is less controlled. DNA in Plasmodium lysates may have a fundamentally shorter stretching factor than DNA in human cell lysates, and measurements from DNA spreads are also skewed to include only the shorter tracts in a population, because longer tracts are more likely to enter a DNA bundle and thus be excluded - a problem that does not arise on long, single, combed DNA fibres. Nevertheless, our measurements are comparable within the conditions used here. The clear result was that DNA replication parameters did not differ significantly in $P$. falciparum versus $P$. knowlesi, although there was a trend towards faster replication fork speeds in P. knowlesi. This trend is corroborated by the observation that in a whole-cell context, briefer pulselabels could be detected in P. knowlesi than in P. falciparum (Supp figure 4).

\section{DISCUSSION}

This study is the first detailed analysis of DNA replication dynamics throughout schizogony in two different malaria parasites, $P$. falciparum and $P$. knowlesi. It reveals some key differences between the two species, such as different periods of active DNA replication and shorter gap phases between successive nuclear replications in $P$. knowlesi than in P. falciparum. There were also similarities: for example, S-phase occupied a similar proportion of the differing cell-cycle lengths in both species.

S-phase occupied $\sim 30 \%$ of each cell cycle, i.e. $15 \mathrm{~h}$ in $P$. falciparum and $9 \mathrm{~h}$ in $P$. knowlesi. During this period, the nuclei in a $P$. knowlesi schizont completed a maximum of 4 and a mean of 2-3 replicative rounds, whereas $P$. falciparum completed a maximum of almost 5 and a mean of 3-4 replicative rounds. $P$. falciparum was therefore proportionally less efficient, averaging an extra $40-$ 45 minutes per nuclear replication. This was primarily due to longer gap phases between replicative rounds, since the genomes are very similar in size ( 23 and $24 \mathrm{Mb}$ ), single-molecule dynamics were not strikingly different in the two species, and active replication periods were not consistently faster in P. knowlesi - rather, they were slower in the first replicative round, and then faster in later schizogony.

There are several explanations for the longer pauses between replicative rounds in $P$. falciparum. This genome may be particularly challenging to replicate, with its high A/T-richness and prevalence of homopolymer tracts leading to errors that require resolution before the chromosomes 
can be separated. If this is true, a 'G2 checkpoint' (well-characterised in mammalian cells but uncharacterised in Plasmodium) must exist. The gap after karyokinesis but before the next replicative round was also longer in $P$. falciparum and this could be imposed, for example, by a certain amount of polymerases or nucleotides being required before a nucleus can trigger replication, and this threshold being slower to achieve in $P$. falciparum than in $P$. knowlesi.

During S-phase the number of genomes in a schizont dramatically increases, but surprisingly there was no set-point in the proportion of genomes that could replicate at once. Schizonts were observed with anything from $20 \%$ to $100 \%$ of their nuclei replicating in a 30 -minute window, with the average proportion of active nuclei being higher in $P$. falciparum than in $P$. knowlesi. Thus, the supply of replication factors must keep pace with the expanding number of genomes - indeed, replicative rounds actually grew faster not slower. The growing anabolic challenge may, however, be one reason why the gaps between replicative rounds increased as schizogony progressed. It may also account for why some nuclei appeared to arrest for much longer than average. A second explanation for arrested nuclei would be unresolvable DNA damage, triggering a checkpoint arrest, although there is little evidence as yet for conventional replication checkpoints in this system.

Both species were strikingly variable in the number of daughter cells produced per schizont. Such variation has been observed repeatedly in $P$. falciparum, with mean merozoite numbers of $15-$ 22 reported in different strains [13, 21-23]. This cannot be entirely due to asynchrony in cultured cells: for example, the majority of $P$. knowlesi cells entered S-phase within a single hour, yet nuclear numbers were extremely variable at $31 \mathrm{hpi}$, when just $2 \mathrm{~h}$ later the great majority of these schizonts would burst. (Asynchronous S-phase entry may play a somewhat greater role in P. falciparum, where the pre-S-phase growth period was $50 \%$ longer and S-phase entry was less tight.) One key explanation for variable schizont size could be heterogenous long-term stalling of replication in some nuclei, as shown in Figure 7: if, at the $2 n$ stage, one nucleus arrests, then the schizont might complete only half as many replicative events and this would logically lead to highly variable schizonts. Another explanation is inherent variability in host erythrocytes, with young or old cells providing more or less hospitable environments for parasite replication. (It was also notable that human erythrocytes from different donors, which can differ in age and many other factors, had a strong impact on cell-cycle length, particularly in $P$. knowlesi, so cell-cycle length can certainly be influenced by host as well as parasite factors.) Regarding parasite factors, different strains of $P$. falciparum are known to have characteristically variable cycle times, ranging from 48h in 3D7 [24] to 42-44h in FCR3 [2]. In this work, only the 3D7 strain was studied.

Our data are largely consistent with data from Ganter and colleagues, who recently characterised S-phase dynamics in P. falciparum in a complementary way, using live-cell microscopy with the replication factor PCNA [9]. They reported that in early schizonts active replication (marked by the presence of PCNA) takes $\sim 40-50$ mins per nucleus, with a broad range in different nuclei and a trend towards longer periods in the first replicative round. The gaps between rounds, both before and after karyokinesis, were much longer that replication itself, and were longer at the 1-to- $2 \mathrm{n}$ stage ( $\sim 75$ mins before and $\sim 50$ mins after karyokinesis) than at the 2 -to- $4 \mathrm{n}$ stage. Cells beyond this stage were not analysed. These data are broadly consistent with ours: that early schizonts tend to take 40-75 minutes per active replicative round and $\sim 3 \mathrm{~h}$ in total between successive nuclear replications. They do not address our additional finding that gaps become longer in mature schizonts, but these authors did generate a mathematical model for overall schizogony, which demanded a slowing of $\sim 17 \%$ in each replicative round after the second. 
It was striking that centrin foci, representing the centriolar plaques that act as microtubule organising centres for each genome division, were only detected with $\sim 50 \%$ of all nuclei in schizonts of either species. This is consistent with detailed microscopy recently published by Guizetti and colleagues [15], and suggests that the centriolar plaques are not static, but are elaborated and disassembled with each replicative round. Furthermore, they clearly disappeared in late schizonts after the final round of karyokinesis: also consistent with Simon et al. [15] and expected if the structures get disassembled when no longer required.

The fact that duplicated centriolar plaques appeared on only a fraction of actively replicating nuclei contradicts the idea that this event initiates DNA replication [2]. In fact, about half of the nuclei that had replicated their DNA within the past 30 minutes showed no detectable centrin foci, and it is not likely that these were all young nuclei that had finished replicating early in the pulselabel and then recently divided, because the putative ' $G 2$ ' gap between the end of replication and karyokinesis was itself at least 30 minutes in half of all events (i.e. when duplicated plaques were visible, only half of those nuclei had been replicating DNA in the past 30 minutes). It is more likely that DNA replication commences before detectable centriolar plaques appear - or at least before their centrin components appear. Furthermore, only a small minority of nuclei showed two wellseparated plaques, suggesting that their duplication and separation is actually a late event in the process of replication and karyokinesis. A caveat here is that centriolar plaques were detected only by centrin, which may be one dynamic component of an underlying, static structure. The detailed organisation of the Plasmodium centriolar plaque remains under active investigation [15].

Although Plasmodium schizogony is a very different process from binary fission in mammalian cells, the intra-nuclear organisation of genome replication appears to be similar. Mammalian nuclei show foci of active replication called 'replication factories'. In early S-phase, when euchromatin is predominantly replicating, factories are small, numerous and dispersed throughout the nucleus; later they appear as larger clumps in the nuclear periphery, representing replicating heterochromatin [25]. Plasmodium nuclei are comparatively tiny but it was nevertheless possible to see brighter foci of newly replicated DNA in the nucleoplasm, resembling replication factories. (These were particularly evident in nuclei stained with $\mathrm{BrdU}$, which was detected less strongly than EdU, using an antibody rather than click-chemistry, thus emphasising the strongest replication foci.) A small proportion of nuclei showed very clear perinuclear foci, possibly because they were captured while replicating discrete perinuclear clusters of heterochromatin, and some showed replication in only one section of the nucleoplasm - these were much commoner in $P$. falciparum than $P$. knowlesi. Chromosomes may be organised differently, or heterochromatin may be more strongly clustered, in $P$. falciparum, reflecting the well-characterised clustered heterochromatin that encodes var virulence genes [26]. Finally, during the presumptive final round of replication, there was a clear pattern of homogenous but faint staining in all nuclei: this was particularly dominant in $P$. falciparum. It suggests that the final round of replication is indeed distinct: replication in all nuclei may slow down, either to ensure synchrony in the final karyokinesis, or because a certain factor becomes limiting. Such low-level DNA synthesis could, alternatively, represent post-replication DNA repair, but this seems unlikely because it was usually seen throughout the nucleoplasm of all nuclei, and quite specifically in late schizonts.

At the single-molecule level, there was little difference in the dynamics of polymerase movement (i.e. replication fork velocity) through the genomes of $P$. falciparum and $P$. knowlesi, although there was a trend towards faster movement in $P$. knowlesi. This could be due to genome composition, or to a higher average level of replication factors (proteins or nucleotides) in $P$. 
knowlesi schizonts. Replication dynamics do frequently differ between different species and even between different human cell lines [27]. Here, however, it seems that the main differences between $P$. falciparum and $P$. knowlesi occur at a higher level - i.e. the timing of replication for whole nuclei and their associated gap phases - rather than at the level of individual replication forks.

Overall, this study provides novel detail about the dynamics of schizogony in two different human malaria parasites, and sets the stage for future work to examine how schizogony changes with changing conditions in the human host. A recent publication reported that nutrient limitation in a murine host can markedly reduce the number of progeny per schizont, and suggested that the same may occur in human malaria patients [28]. This may be only one of many host conditions that could influence the growth of malaria parasites, with potentially important impacts on clinical outcome.

\section{ACKNOWLEDGEMENTS}

We are grateful to the lab of Prof. Julian Rayner for making the initial thymidine kinase transfectant in P. knowlesi, and to Dr Francis Totanes and Dr Andrew Blagborough for critical reading of the manuscript.

\section{METHODS}

\section{Parasite culture and transfection for ectopic expression of thymidine kinase}

P. falciparum parasites were maintained in vitro in human O+ erythrocytes at $4 \%$ haematocrit in RPMI 1640 medium supplemented with 25mM HEPES (Sigma-Aldrich), 0.25\% sodium bicarbonate, $50 \mathrm{mg} / \mathrm{L}$ hypoxanthine standard procedures [29]. P. knowlesi parasites were maintained similarly, maintained at $2 \%$ haematocrit instead of $4 \%$ and supplemented with $22.2 \mathrm{mM}$ glucose and $10 \%$ horse serum instead of human serum.

The $P$. falciparum 3D7 strain that expresses thymidine kinase has been previously described [8]. A similar thymidine-kinase-expressing $P$. knowlesi strain was created by transfecting the same plasmid into the A1-H.1 strain, essentially as previously described [30]. Late-stage $P$. knowlesi parasites were enriched using Histodenz and $10 \mu \mathrm{l}$ of schizonts mixed in a transfection cuvette (Lonza) with $100 \mu \mathrm{l}$ of P3 solution (Lonza) containing $30 \mu \mathrm{g}$ of plasmid. Transfection was carried out using program FP158 (Amaxa Nucleofector, Lonza), followed by immediate transfer into $500 \mu \mathrm{l}$ of complete culture media mixed with $190 \mu$ l uninfected erythrocytes. The transfection mix was incubated at $37^{\circ} \mathrm{C}$ while shaking at $800 \mathrm{rpm}$ in a thermomixer for $30 \mathrm{mins}$, before being transferred into a 6-well plate, gassed and incubated for one parasite life cycle. Selection was then applied with $100 \mathrm{nM}$ pyrimethamine (Santa Cruz Biotechnology Inc) and daily media changes for 3 days, then routine maintenance until transgenic parasites appeared

\section{Synchronisation for timecourse experiments}

Mature schizont cultures at $>6 \%$ parasitaemia were synchronised using $55 \%$ Nycodenz (Alere technologies AS). Cultures were centrifuged and media removed to leave $2 \mathrm{ml}$ of media and blood, which was layered gently on top of $5 \mathrm{~mL}$ of prewarmed $55 \%$ Nycodenz, then centrifuged at $1300 \mathrm{~g}$ for 5 mins. The floating schizont layer was collected and added to a wash buffer containing incomplete RPMI, 4\% haematocrit for the final culture volume, and $1.5 \mu \mathrm{M}$ 'Compound 2' (4-[7[(dimethylamino)methyl]-2-(4-fluorophenyl)imidazo[1,2-a]pyridine-3-yl]pyrimidin-2-amine) [31]), 
centrifuged ( $800 \mathrm{~g}$ for 5 mins), resuspended in complete RPMI and $1.5 \mu \mathrm{M}$ Compound 2 and incubated at $37^{\circ} \mathrm{C}$ for $2 \mathrm{~h}$. Cultures were centrifuged (800xg for $5 \mathrm{mins}$ ) and supernatant removed. Cultures were washed in prewarmed incomplete media and resuspended in complete RPMI to allow reinvasion. Cultures were split into $5 \mathrm{ml}$ aliquots in $50 \mathrm{ml}$ falcon tubes and placed in an orbital shaker at $37^{\circ} \mathrm{C}$ for $1 \mathrm{~h}$ to increase reinvasion rate. After reinvasion, the cultures were pooled together, centrifuged and Nycodenz treated again, now retaining the bottom layer containing newly reinvaded ring stages. This layer was washed in incomplete media and resuspended in compete RPMI, marking timepoint 0 hours post invasion (hpi).

\section{Pulse labelling with modified nucleotides}

Cultures for single-labelled timecourses were pulse-labelled with $10 \mu \mathrm{M}$ ethyl-deoxyuridine (EdU) for 30 mins at $1 \mathrm{~h}$ intervals. Cultures for double-labelling experiments were first pulse-labelled with 10 $\mu \mathrm{M}$ EdU for 30 mins at specific timepoints, then washed twice in prewarmed incomplete RPMI and resuspended in complete media that was incubated alongside the cultures throughout the experiments, to avoid any disturbance of cell cycle dynamics caused by switching into fresh media. At specific timepoints, cultures were labelled with a second nucleotide, 5-bromo-2'-deoxyuridine (BrdU), at $200 \mu \mathrm{M}$ for 30 mins at $37^{\circ} \mathrm{C}$. Immediately after this label, blood smears were made, air dried, fixed in $2 \%$ paraformaldehyde for 5 mins, washed in PBS, washed in $\mathrm{dH}_{2} \mathrm{O}$, air dried and stored at $4^{\circ} \mathrm{C}$.

\section{Immunofluorescence}

All slides were incubated in $0.2 \%$ Triton X-100 for 15 mins and washed in PBS for 5 mins.

Single-nucleotide EdU-labelled slides were incubated in blocking solution (1\% BSA in PBS) for 30 mins and then 1:100 anti-centrin antibody (clone 20H5, Millipore) in blocking solution for $1 \mathrm{~h}$ at room temperature. Slides were washed three times in blocking solution. EdU signal was detected with click chemistry. Slides were incubated in click reaction buffer $(0.845 \mathrm{mM}$ Tris $\mathrm{HCl}$ pH $8.8,1 \mathrm{mM}$ $\mathrm{CuSO}_{4}, 2.5 \mu \mathrm{M}$ Alexa Fluorescent Azide 594, freshly dissolved $75 \mathrm{mM}$ ascorbic acid) for $1 \mathrm{~h}$ at room temperature. Slides were washed in blocking solution three times and then incubated with the secondary antibody for centrin detection, goat anti-mouse Alexa 488 (Molecular Probes) for $1 \mathrm{~h}$ at room temperature. Slides were washed twice in PBS, incubated with $2 \mu \mathrm{g} / \mathrm{ml}$ 4],6-diamidino-2phenylindole (DAPI) for 10 mins, washed in PBS, mounted using $20 \mu \mathrm{l}$ Prolong Diamond Antifade (Molecular Probes) and set overnight at room temperature.

Double-labelled EdU/BrdU slides were incubated with $0.2 \%$ Triton- $X$ for 15 mins, washed in PBS for 5 mins, incubated in $1 \mathrm{M} \mathrm{HCl}$ for $1 \mathrm{~h}$ and washed in PBS for 5 mins. EdU labels were "clicked" as above. Slides were washed with PBS three times. Slides were then incubated with $20 \mathrm{mM}$ nonfluorescent dye Azidomethylphenosulphide (Sigma) for 30 mins at room temperature to block remaining EdU residues. Slides were incubated with blocking solution for 30 mins. Primary immunodetection of BrdU was with rat anti BrdU BU1/75 (ICR1) antibody (1:100 dilution, Abcam). Slides were washed in blocking solution three times and incubated with goat anti-rat Alexa 488 secondary antibody (1:500 dilution, Molecular probes) at room temperature for $1 \mathrm{~h}$; washed, incubated with DAPI and mounted as above.

\section{Data analysis and statistics}

100 parasites were counted for each timepoint of the single-nucleotide-labelled timecourses, and 50 parasites were counted for double-labelled timecourses. For the 'stalling' experiment, 20 parasites 
were counted for each category of schizont maturity. Images were classified regarding presence and number of centrin foci and the presence and pattern of nucleotide labelling ( $\mathrm{BrdU} / \mathrm{EdU})$ within the nucleus. Data were plotted using Graphpad Prism and the statistical significance of differences between groups of data was calculated via Mann Whitney tests or analysis of variance.

\section{DNA fibre spreading}

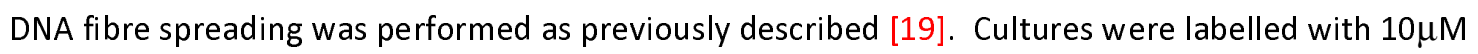
EdU for 10 mins, then $100 \mu \mathrm{M}$ BrdU for 10 mins. $2 \mu \mathrm{l}$ of saponin-released parasites was pipetted near the top of a glass slide and allowed to dry for $\sim 5$ mins, until sticky but not dry. $7 \mu$ l of spreading buffer ( $20 \mathrm{mM}$ TrisHCl pH 7.4, $50 \mathrm{mM}$ EDTA pH 8, 0.5\% SDS) was added and stirred gently with a pipette tip to release the DNA. The slide was incubated for 2 mins and then tilted $\left(15^{\circ}\right)$ to let drop run slowly down the slide, producing a constant stretching factor of $2.59 \mathrm{~kb} / \mu \mathrm{M}$ [20]. Slides were air dried, fixed in $\mathrm{MeOH}$ : acetic acid (3:1), air dried and stored at $4{ }^{\circ} \mathrm{C}$.

\section{Detection of EdU and BrdU in DNA fibre spreads}

Fibre spreads were denatured with $1 \mathrm{M} \mathrm{HCl}$ for 75 mins, washed three times in PBS and blocked with blocking solution (1\% BSA and $0.1 \%$ Tween 20 in PBS). Immuno-detection of double labelled fibre spreads was done with EdU click chemistry and then antibodies diluted in blocking solution, each incubated with a coverslip on top in a humid chamber at room temperature for $1 \mathrm{~h}$. Slides were incubated with click reaction buffer as above, followed with three washes in blocking buffer. Slides were then incubated in blocking solution for 30 mins. Primary immuno-detection for BrdU was done with rat anti BrdU BU1/75 (ICR1) antibody (1:100 dilution, Abcam), together with mouse anti ssDNA (clone16-19) antibody (Millipore, 1:300 dilution). The secondary antibodies (Molecular Probes) were goat anti-rat coupled to Alexa 488 (1:500 dilution) and goat anti-mouse coupled to Alexa 405 (1:500 dilution). Slides were washed three times in PBS and mounted using $20 \mu \mathrm{l}$ Prolong Diamond Antifade (Molecular Probes), set overnight at room temperature. Single-labelled fibre spreads were detected above but without click chemistry.

\section{Image Acquisition and Processing of DNA fibre data}

Image acquisition was via a Nikon Microphot SA microscope equipped with a Qimaging Retiga R6 camera. Images were acquired with a $100 \mathrm{X}$ oil objective where $1 \mu \mathrm{m}=28.65$ pixels, which corresponds to $69.8 \mathrm{bp}$ per pixel (DNA stretching factor $2 \mathrm{~kb} / \mu \mathrm{m}$ for DNA combing) and $90.40 \mathrm{bp}$ per pixel (DNA stretching factor $2.59 \mathrm{~kb} / \mu \mathrm{m}$ for DNA spreading). Observation of long DNA fibres required the capture and assembly of adjacent fields. Replication tracts and fibre lengths were measured manually using ImageJ software. Statistical analysis and graphs of BrdU tract length and replication velocities were performed using GraphPad Prism.

\section{REFERENCES}

1. Striepen B, Jordan CN, Reiff S, van Dooren GG. Building the perfect parasite: cell division in apicomplexa. PLoS pathogens. 2007;3(6):e78. doi: 10.1371/journal.ppat.0030078. PubMed PMID: 17604449; PubMed Central PMCID: PMCPMC1904476.

2. Arnot DE, Ronander E, Bengtsson DC. The progression of the intra-erythrocytic cell cycle of Plasmodium falciparum and the role of the centriolar plaques in asynchronous mitotic division 
during schizogony. International journal for parasitology. 2011;41(1):71-80. doi: 10.1016/j.ijpara.2010.07.012. PubMed PMID: 20816844.

3. Wassmer SC, Taylor TE, Rathod PK, Mishra SK, Mohanty S, Arevalo-Herrera M, et al. Investigating the Pathogenesis of Severe Malaria: A Multidisciplinary and Cross-Geographical Approach. Am J Trop Med Hyg. 2015. doi: 10.4269/ajtmh.14-0841. PubMed PMID: 26259939.

4. Dondorp AM, Desakorn V, Pongtavornpinyo W, Sahassananda D, Silamut K, Chotivanich K, et al. Estimation of the total parasite biomass in acute falciparum malaria from plasma PfHRP2. PLoS Med. 2005;2(8):e204. doi: 10.1371/journal.pmed.0020204. PubMed PMID: 16104831; PubMed Central PMCID: PMCPMC1188247.

5. Branco A, Melo-Cristino J. Extreme parasitemia in P falciparum malaria. Blood. 2018;132(8):868. doi: 10.1182/blood-2018-07-861880. PubMed PMID: 30139832.

6. Gardner MJ, Hall N, Fung E, White O, Berriman M, Hyman RW, et al. Genome sequence of the human malaria parasite Plasmodium falciparum. Nature. 2002;419(6906):498-511. PubMed PMID: 12368864.

7. Stanojcic S, Kuk N, Ullah I, Sterkers Y, Merrick CJ. Single-molecule analysis reveals that DNA replication dynamics vary across the course of schizogony in the malaria parasite Plasmodium falciparum. Sci Rep. 2017;7(1):4003. doi: 10.1038/s41598-017-04407-z. PubMed PMID: 28638076; PubMed Central PMCID: PMCPMC5479783.

8. Merrick CJ. Transfection with thymidine kinase permits bromodeoxyuridine labelling of DNA replication in the human malaria parasite Plasmodium falciparum. Malar J. 2015;14(490). doi: 10.1186/s12936-015-1014-7.

9. Klaus SB, P.; Kim, J.; Machado, M.; Funaya, C.; Schaaf, V.; Klaschka, D.; Kudulyte, A.; Cyrklaff, M.; Laketa, V.; Höfer, T.; Guizetti, J.; Becker, N.B.; Frischknecht, F.; Schwarz, U.S.; Ganter, M. Asynchronous nuclear cycles in multinucleated Plasmodium falciparum enable rapid proliferation. BioRxiv. 2021;doi.org/10.1101/2021.04.15.440016

10. Arnot DE, Gull K. The Plasmodium cell-cycle: facts and questions. Annals of tropical medicine and parasitology. 1998;92(4):361-5. PubMed PMID: 9683889.

11. Gerald N, Mahajan B, Kumar S. Mitosis in the human malaria parasite Plasmodium falciparum. Eukaryotic cell. 2011;10(4):474-82. doi: 10.1128/EC.00314-10. PubMed PMID: 21317311; PubMed Central PMCID: PMCPMC3127633.

12. Francia ME, Striepen B. Cell division in apicomplexan parasites. Nat Rev Microbiol. 2014;12(2):125-36. doi: 10.1038/nrmicro3184. PubMed PMID: 24384598.

13. Reilly HB, Wang H, Steuter JA, Marx AM, Ferdig MT. Quantitative dissection of clone-specific growth rates in cultured malaria parasites. International journal for parasitology. 2007;37(14):1599607. doi: 10.1016/j.ijpara.2007.05.003. PubMed PMID: 17585919; PubMed Central PMCID: PMCPMC2268714.

14. Rudlaff RM, Kraemer S, Marshman J, Dvorin JD. Three-dimensional ultrastructure of Plasmodium falciparum throughout cytokinesis. PLoS pathogens. 2020;16(6):e1008587. doi: 10.1371/journal.ppat.1008587. PubMed PMID: 32511279; PubMed Central PMCID: PMCPMC7302870.

15. Simon CS, Funaya C, Bauer J, Vobeta Y, Machado M, Penning A, et al. An extended DNA-free intranuclear compartment organizes centrosome microtubules in malaria parasites. Life Sci Alliance. 2021;4(11). doi: 10.26508/Isa.202101199. PubMed PMID: 34535568; PubMed Central PMCID: PMCPMC8473725. 
16. Michalet X, Ekong R, Fougerousse F, Rousseaux S, Schurra C, Hornigold N, et al. Dynamic molecular combing: stretching the whole human genome for high-resolution studies. Science (New York, NY. 1997;277(5331):1518-23. PubMed PMID: 9278517.

17. Lebofsky R, Bensimon A. Single DNA molecule analysis: applications of molecular combing. Brief Funct Genomic Proteomic. 2003;1(4):385-96. PubMed PMID: 15239885.

18. Schwob E, de Renty C, Coulon V, Gostan T, Boyer C, Camet-Gabut L, et al. Use of DNA combing for studying DNA replication in vivo in yeast and mammalian cells. Methods Mol Biol. 2009;521:673-87. PubMed PMID: 19563133.

19. Parra I, Windle B. High resolution visual mapping of stretched DNA by fluorescent hybridization. Nature genetics. 1993;5(1):17-21. doi: 10.1038/ng0993-17. PubMed PMID: 8106079. 20. Jackson DA, Pombo A. Replicon clusters are stable units of chromosome structure: evidence that nuclear organization contributes to the efficient activation and propagation of $S$ phase in human cells. The Journal of cell biology. 1998;140(6):1285-95. PubMed PMID: 9508763; PubMed Central PMCID: PMC2132671.

21. Garg S, Agarwal S, Dabral S, Kumar N, Sehrawat S, Singh S. Visualization and quantification of Plasmodium falciparum intraerythrocytic merozoites. Syst Synth Biol. 2015;9(Suppl 1):23-6. doi: 10.1007/s11693-015-9167-9. PubMed PMID: 26702305; PubMed Central PMCID: PMCPMC4688406. 22. Simon CS, Sturmer VS, Guizetti J. How Many Is Enough? - Challenges of Multinucleated Cell Division in Malaria Parasites. Front Cell Infect Microbiol. 2021;11:658616. doi:

10.3389/fcimb.2021.658616. PubMed PMID: 34026661; PubMed Central PMCID: PMCPMC8137892.

23. Stewart LB, Diaz-Ingelmo O, Claessens A, Abugri J, Pearson RD, Goncalves S, et al. Intrinsic multiplication rate variation and plasticity of human blood stage malaria parasites. Commun Biol. 2020;3(1):624. doi: 10.1038/s42003-020-01349-7. PubMed PMID: 33116247; PubMed Central PMCID: PMCPMC7595149.

24. Bell DR, S.; Patel, A.P.; Lee, S.H.; Chang, Y.T.; Deu, E. High Content and High Throughout Phenotypic Assay for the Hourly Resolution of the Malaria Parasite Erythrocytic Cycle. BioRxiv. 2021; doi.org/10.1101/2021.03.15.434938 Epub 2021.

25. Dimitrova DS, Gilbert DM. Temporally coordinated assembly and disassembly of replication factories in the absence of DNA synthesis. Nat Cell Biol. 2000;2(10):686-94. doi: 10.1038/35036309. PubMed PMID: 11025658; PubMed Central PMCID: PMC1255923.

26. Freitas-Junior LH, Bottius E, Pirrit LA, Deitsch KW, Scheidig C, Guinet F, et al. Frequent ectopic recombination of virulence factor genes in telomeric chromosome clusters of $P$. falciparum. Nature. 2000;407(6807):1018-22. PubMed PMID: 11069183.

27. Techer H, Koundrioukoff S, Azar D, Wilhelm T, Carignon S, Brison O, et al. Replication dynamics: biases and robustness of DNA fiber analysis. Journal of molecular biology. 2013;425(23):4845-55. doi: 10.1016/j.jmb.2013.03.040. PubMed PMID: 23557832.

28. Mancio-Silva L, Slavic K, Grilo Ruivo MT, Grosso AR, Modrzynska KK, Vera IM, et al. Nutrient sensing modulates malaria parasite virulence. Nature. 2017;547(7662):213-6. doi: 10.1038/nature23009. PubMed PMID: 28678779; PubMed Central PMCID: PMCPMC5511512.

29. Trager W, Jensen JB. Human malaria parasites in continuous culture. Science (New York, NY. 1976;193(4254):673-5. PubMed PMID: 781840.

30. Moon RW, Hall J, Rangkuti F, Ho YS, Almond N, Mitchell GH, et al. Adaptation of the genetically tractable malaria pathogen Plasmodium knowlesi to continuous culture in human erythrocytes. Proceedings of the National Academy of Sciences of the United States of America. 
2013;110(2):531-6. doi: 10.1073/pnas.1216457110. PubMed PMID: 23267069; PubMed Central PMCID: PMCPMC3545754.

31. Donald RG, Zhong T, Wiersma H, Nare B, Yao D, Lee A, et al. Anticoccidial kinase inhibitors: identification of protein kinase targets secondary to CGMP-dependent protein kinase. Molecular and biochemical parasitology. 2006;149(1):86-98. doi: 10.1016/j.molbiopara.2006.05.003. PubMed PMID: 16765465.

\section{FIGURE LEGENDS}

\section{Figure 1: Schematics of experimental setup}

A: Schematics of timecourses using single EdU pulse labels.

B: Schematic of experiments using sequential pulses of two nucleotides ( $E d U$ and $B r d U$ ) to determine the length of each replicative round.

C: Schematics of timecourses using 2-nucleotide ( $E d U$ and BrdU) interval pulse labels.

\section{Figure 2: Length and dynamics of S-phase in $P$. falciparum and $P$. knowlesi}

A, B: Scatter graphs of nuclear numbers at each hour in P. falciparum (A) or $P$. knowlesi (B), $n=100$ cells, medians are shown in red.

C, D: Percentage of 100 cells showing some EdU labelling at each hour in $P$. falciparum (C) and $P$. knowlesi (D).

E, F: Percentage of replicating nuclei per cell at each timepoint in P. falciparum (E) and P. knowlesi $(F), n=100$, medians are shown in red.

$G, H$ : Percentage of replicating nuclei per cell, re-plotted by number of nuclei, in P. falciparum (G) and $P$. knowlesi $(\mathrm{H}), \mathrm{n}=100$, medians are shown in red.

\section{Figure 3: Patterns of replication within nuclei in $P$. falciparum and $P$. knowlesi}

A: Examples of 5 distinct patterns of EdU incorporation seen in replicating nuclei. Scale bar $1 \mu \mathrm{m}$.

B: Representative examples of cells at each hour across the timecourse for $P$. falciparum and $P$. knowlesi. Scale bar $2 \mu \mathrm{m}$.

C: Percentages of each distinct pattern seen at each point across the timecourse in P. falciparum and

P. knowlesi.

D: Intensity of EdU staining (calculated as 'integrated density' across each nucleus) seen at each timepoint in $P$. falciparum and $P$. knowlesi. A significant drop in EdU staining intensity is seen at the end of the timecourse in both species ( ${ }^{* * *}, \mathrm{p}=0.001$, ANOVA). In $P$. falciparum, this correlates clearly with the rise at $44-47 \mathrm{hpi}$ in the pattern qualitatively categorised as 'less bright' in (C).

\section{Figure 4: Relationship between centriolar plaques and replicating nuclei}

A: Schematic showing the process of karyokinesis that has previously been proposed in Plasmodium, highlighting the role of the centriolar plaque (adapted from Gerald et al. [11]).

B: Examples of the distinct patterns of centrin staining seen on the highlighted nuclei: no foci, a single focus, 2 adjacent foci, 2 opposite foci. Scale bar $1 \mu \mathrm{m}$.

C: Percentage of nuclei with 0,1 or 2 centrin foci throughout schizogony, $n=100$.

D: Percentage of nuclei with 0,1 or 2 centrin foci that also showed or did not show active DNA replication (EdU staining) within the previous 30 mins.

Figure 5: Replicative rounds become faster across the course of schizogony 
A: Representative examples of $P$. falciparum cells labelled with EdU for 30 minutes and then a 15minute pulse of $\mathrm{BrdU}$ at increasing intervals. Scale bar $2 \mu \mathrm{m}$.

B: Graphs showing the percentage of all nuclei that labelled with EdU and BrdU, firstly in mononuclear cells and then for $2-n, 3-6 n$ and $>6-n$ cells $(n=20)$. Scatter plots display the same data broken down per-cell.

C: Representative examples of $P$. knowlesi cells labelled with EdU for 30 minutes and then a 15minute pulse of $\mathrm{BrdU}$ at increasing intervals. Scale bar $2 \mu \mathrm{m}$.

D: Graphs as in (B) showing the percentage of nuclei labelled with EdU and BrdU in P. knowlesi cells of increasing ploidy ( $n=20$ per category).

Figure 6: Gap phases lengthen across the course of schizogony

A,C: Representative examples of cells across double-labelled timecourses for $P$. falciparum (A) and $P$. knowlesi (C). P. falciparum was labelled at $3 \mathrm{~h}$ intervals, up a maximum of $9 \mathrm{~h}$ (35-44hpi); $P$. knowlesi at $2 \mathrm{~h}$ intervals, up a maximum of $10 \mathrm{~h}$ (18-28hpi). Scale bars $2 \mu \mathrm{m}$.

B,D: Percentages of nuclei per cell labelled with EdU and BrdU, EdU alone, or BrdU alone throughout each timecourse. Dotted lines separate the datasets from different time-intervals.

Figure 7: Long-term arrest of individual nuclei is common during schizogony

A: Representative examples of $P$. falciparum cells double-labelled with EdU for 30 minutes and then BrdU for 5 h. Scale bar $2 \mu \mathrm{m}$.

B: Percentages of all nuclei labelled with EdU and BrdU, EdU alone, BrdU alone, or neither label ( $n=$ 20 cells, stratified into cells with up to 6 nuclei or more than 6 nuclei).

C: Scatter plots showing percentages of nuclei per cell with the various labelling patterns.

D: Representative examples of $P$. knowlesi cells double-labelled with EdU for 30 minutes and then BrdU for $5 \mathrm{~h}$. Scale bar $2 \mu \mathrm{m}$.

E: Percentages of all nuclei labelled with EdU and BrdU, EdU alone, BrdU alone, or neither label $(n=$ 20 cells, stratified into cells with up to 6 nuclei or more than 6 nuclei).

F: Scatter plots showing percentages of nuclei per cell with the various labelling patterns.

Figure 8: Single-molecule DNA replication dynamics in P. falciparum and $P$. knowlesi

A: Schematic showing possible patterns of labelling on DNA fibres. Pattern 1 is an ongoing replication fork, moving throughout the two consecutive pulse-labels. Pattern 2 shows origins that fired during the first and second pulse labels, with inter-origin distance (IOD) measured as centre to centre distance between two adjacent origins. Pattern 3 shows two replication forks terminating during the first or second pulses. The presumed positions of the replication origins are indicated with asterisks in the middle of bidirectional replication forks. Arrows represent the direction of the replication forks' progression.

B: Representative DNA fibre spreads from $P$. falciparum and $P$. knowlesi. A $5 \mathrm{~kb}$ scale bars is indicated.

C: Dot plot showing distribution of replication fork speeds in $P$. falciparum and $P$. knowlesi, calculated on the basis of a $20-\mathrm{min}$ pulse label (10 mins EdU, 10mins BrdU) and a stretching factor of $2.59 \mathrm{~kb} / \mu \mathrm{m}$. Red bars represent the median value.

D: Dot plot showing distribution of inter-origin distances (IODs) in P. falciparum and P. knowlesi, calculated on the basis of a stretching factor of $2.59 \mathrm{~kb} / \mu \mathrm{m}$. Red bars represent the median value. 


\section{Supplementary figure 1}

Example of a typical slide, showing 3 parasites classified for their number of nuclei, number of centrin foci and presence/absence of EdU staining.

\section{Supplementary figure 2}

A: P. falciparum scatter graphs of nuclear numbers at each point of the double--labelled labelled timecourse shown in main Figure 6.

B: $P$. knowlesi scatter graphs of nuclear numbers every 2 hours across the double-labelled timecourse shown in main Figure 6.

Final timepoints show reinvasion.

\section{Supplementary figure 3}

A: Percentage of nuclei with 0,1 or 2 centrin foci and patterns of intranuclear DNA replication (full, partial, or discrete foci) throughout schizogony.

B: Data as in Data as in A, replotted by number of by number of nuclei per cell rather than hpi.

\section{Supplementary figure 4}

The minimum pulse-labelling period that can be detected via EdU--labelled DNA was tested. In $P$. falciparum, 3 minutes was detectable (faintly) and 5 minutes gave reasonably bright signal. In $P$. knowlesi, labelling was clearly detectable within 1 within 1 minute.

\section{Supplementary figure 5}

A: Representative examples of cells across the double-labelled timecourse with $2 \mathrm{~h}$ intervals for $P$. falciparum. Scale bar $2 \mu \mathrm{m}$.

B: Percentages of nuclei labelled with EdU alone, BrdU alone, or both labels throughout the timecourses shown in (A).

\section{Supplementary figure 6}

Data as in main Figure 7, showing the percentages of $P$. knowlesi nuclei labelled with $\mathrm{EdU}$ and $\mathrm{BrdU}$, EdU alone, BrdU alone, or neither label ( $n=20$ cells), after both $2 \mathrm{~h}$ and $5 \mathrm{~h}-$ demonstrating a higher percentage of arrested nuclei after $2 \mathrm{~h}$ ( $22 \%$ of S-phase) than $5 \mathrm{~h}$ ( $>50 \%$ of S-phase), but an overall similar picture. Data are also stratified into cells with 2-3, 4- 6, or more than 6 nuclei, showing that arrested nuclei are still detected in very young $2-3 n$ schizonts. 
Fig 1

A $*$ Addition of EdU

\section{P. falciparum}

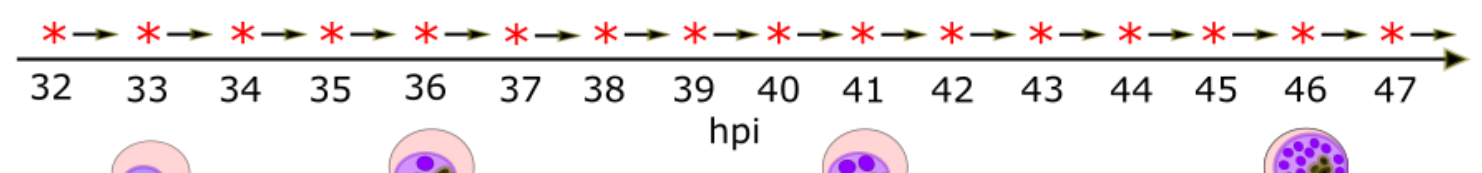

(8.)

\section{P. knowlesi}

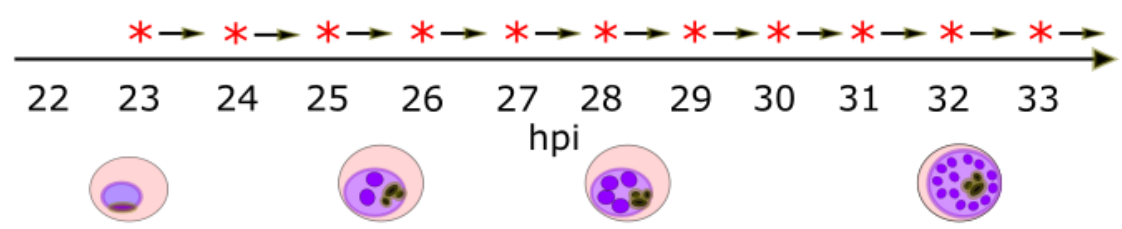

B

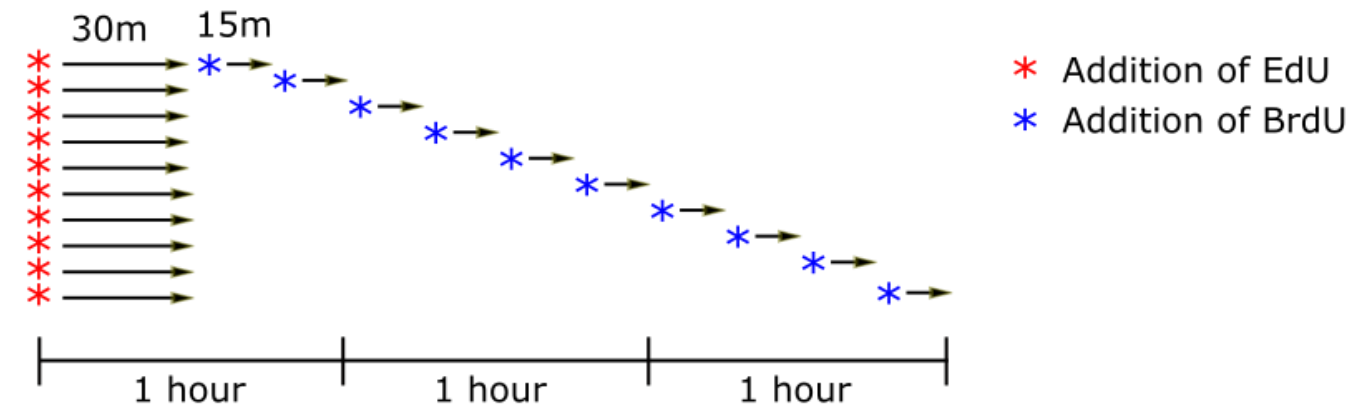

C

\section{P. falciparum}

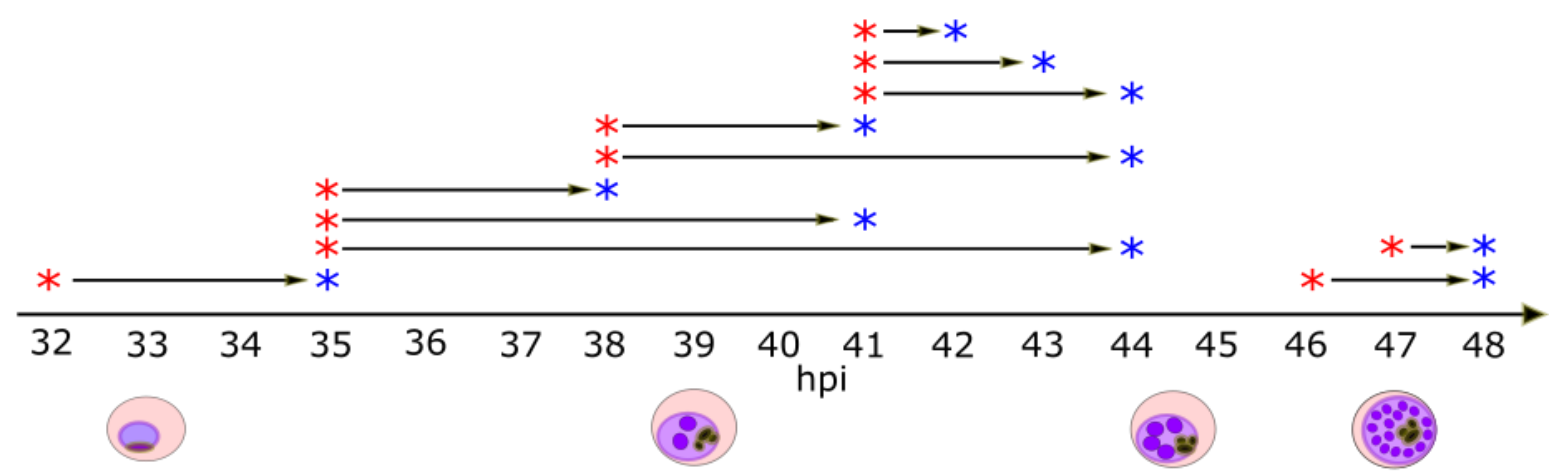

P. knowlesi

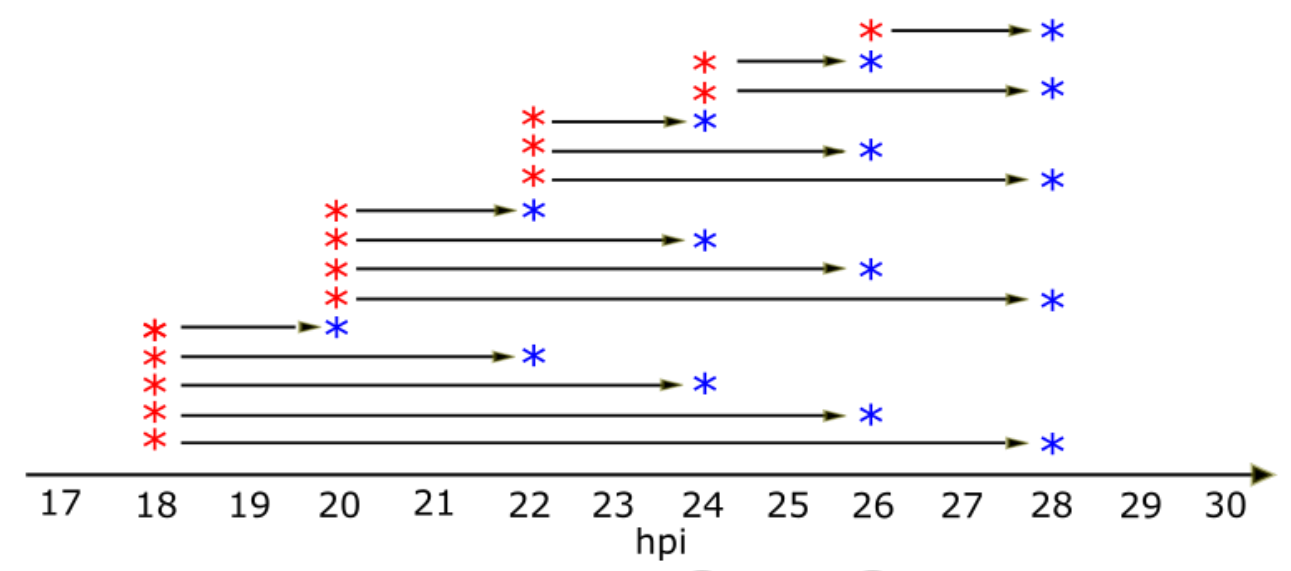


A

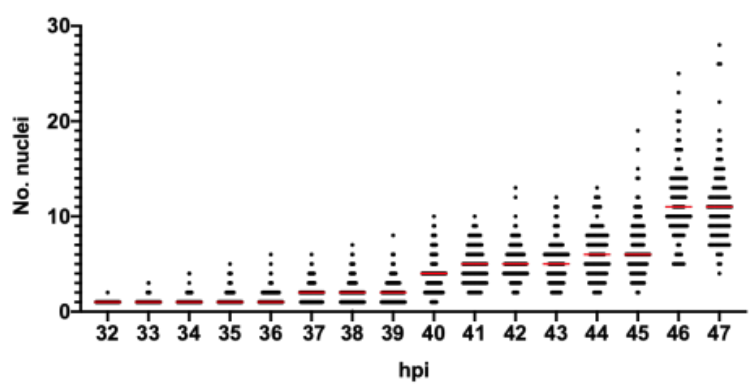

C

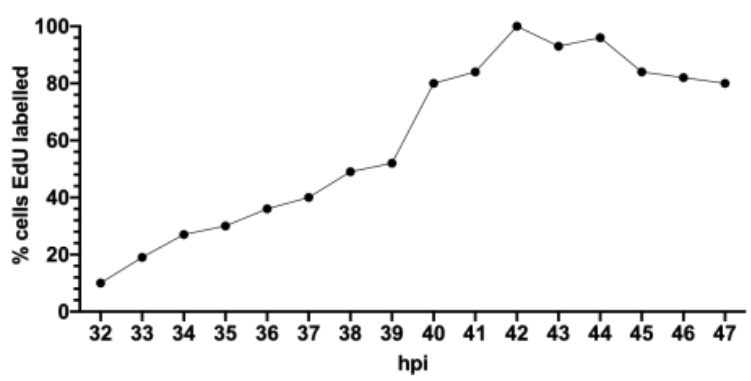

E

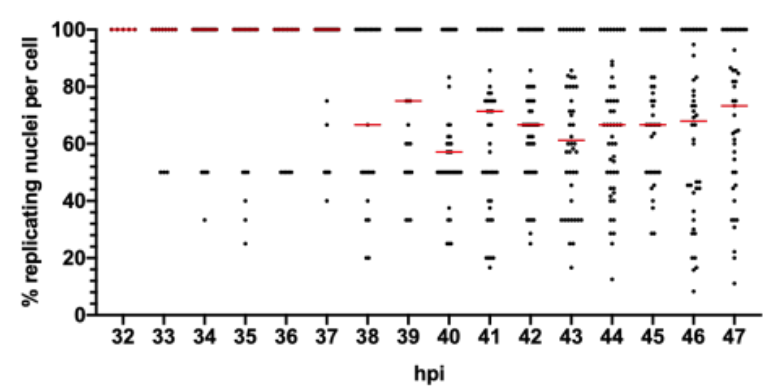

G

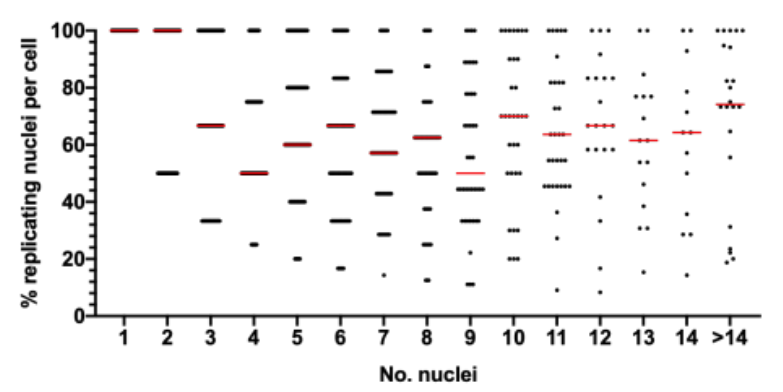

B

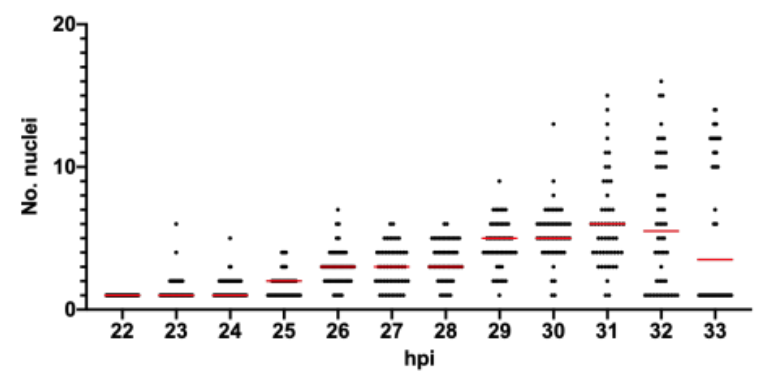

D

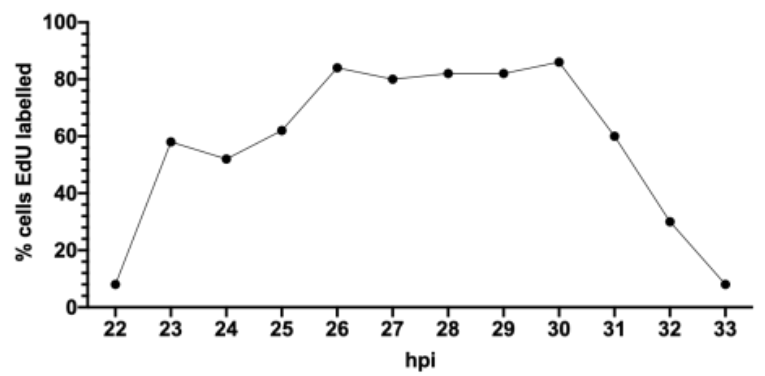

F

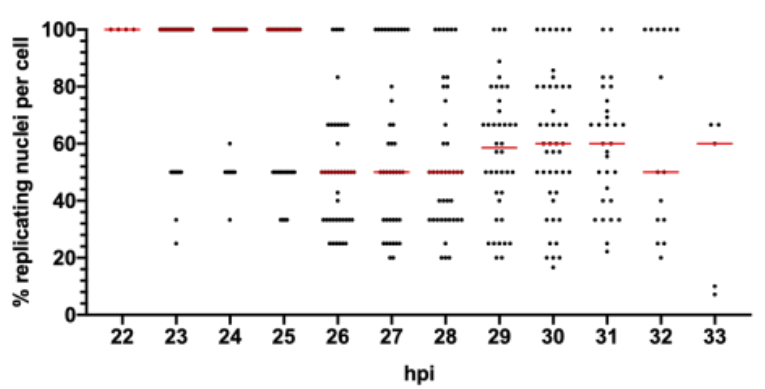

$\mathrm{H}$

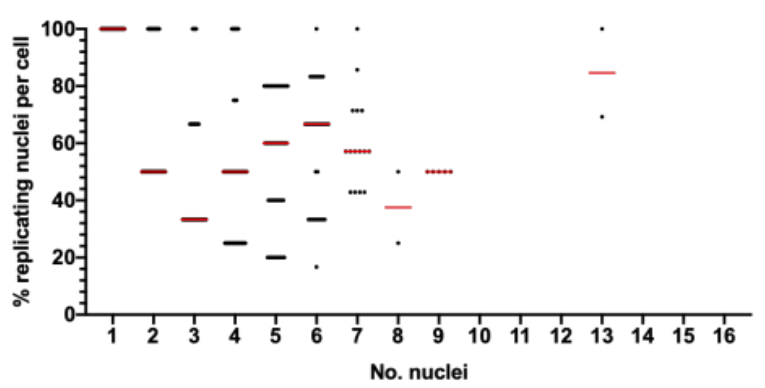


Fig 3

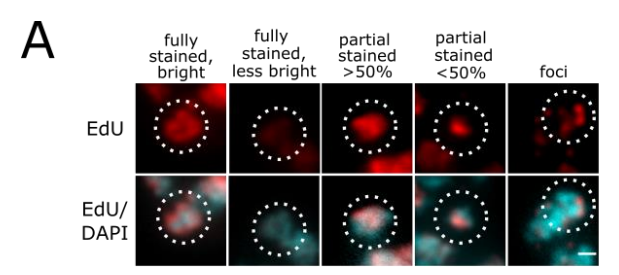

B
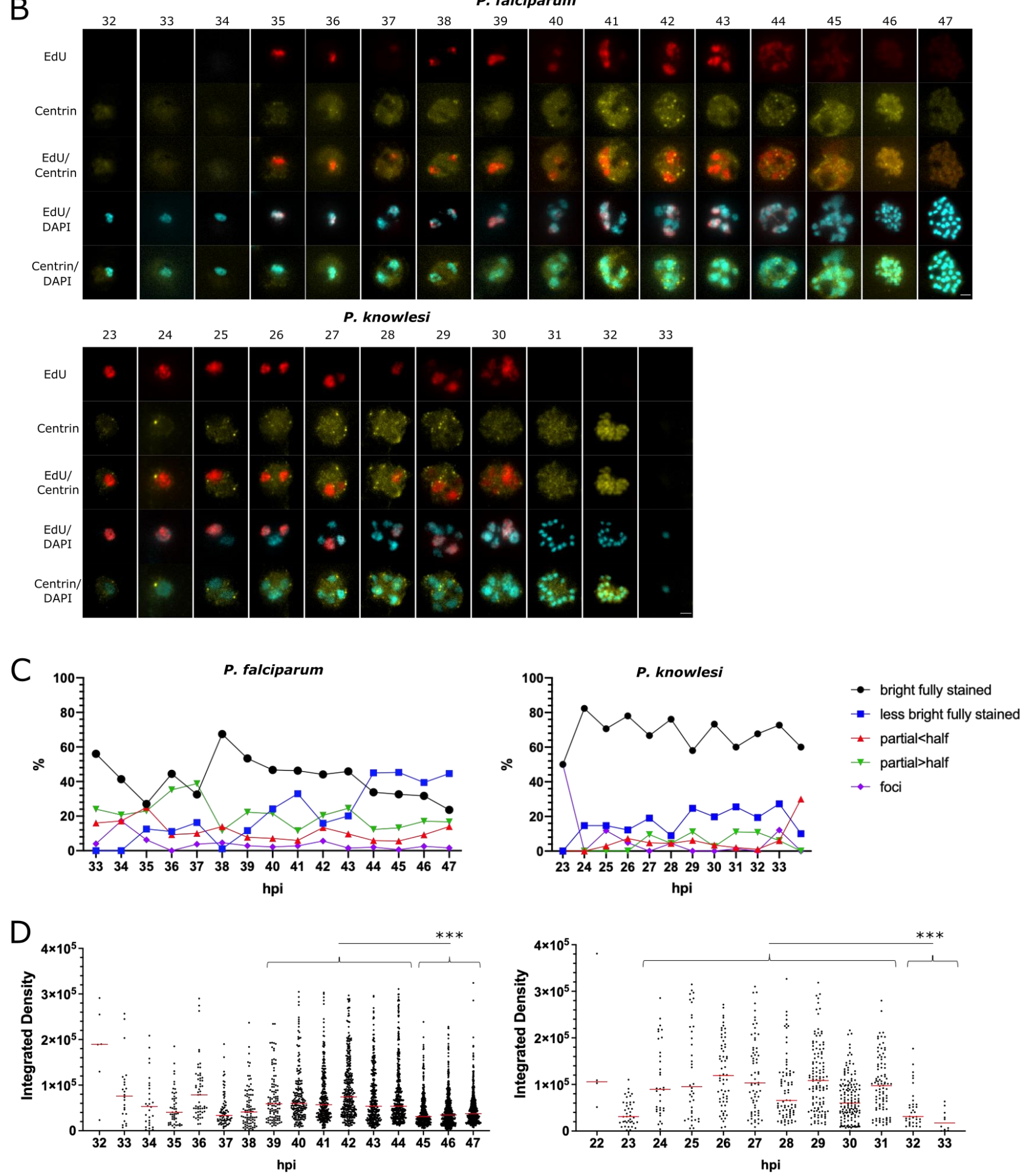


\section{Fig 4}

A

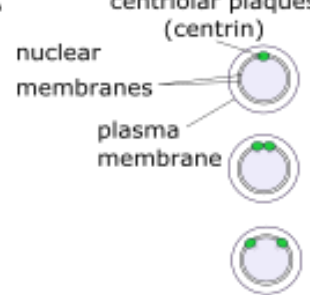

microtubules

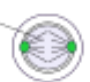

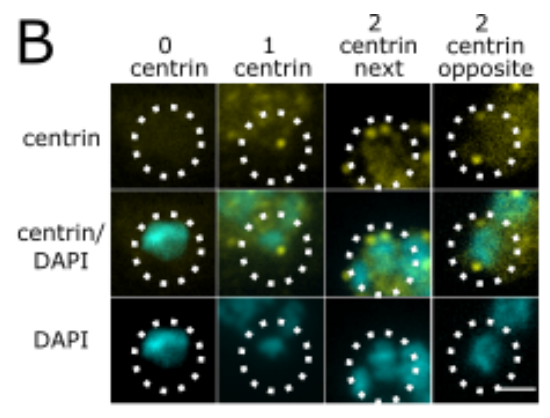

C

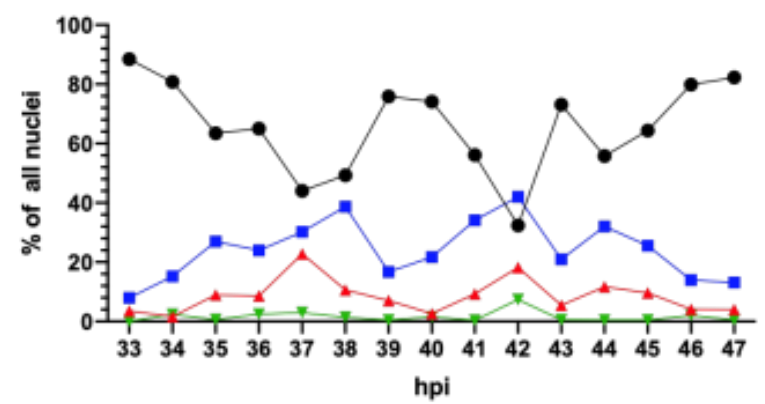

P. falciparum

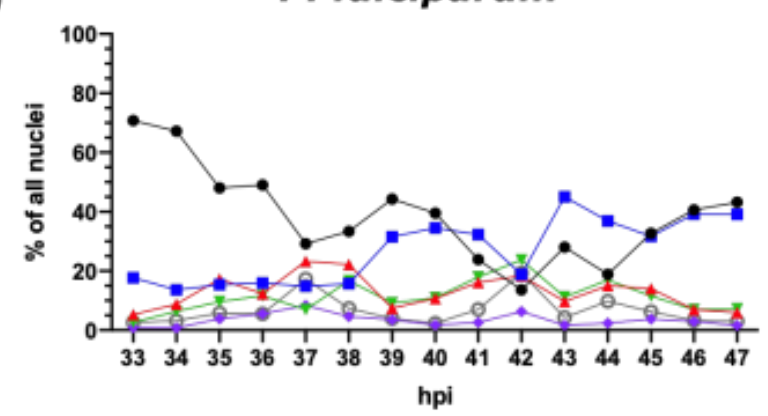

P. knowlesi

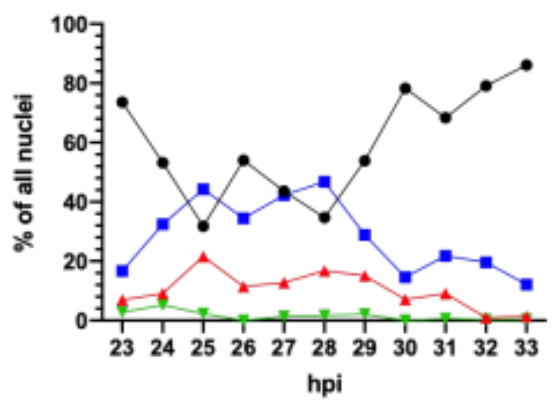

P. knowlesi

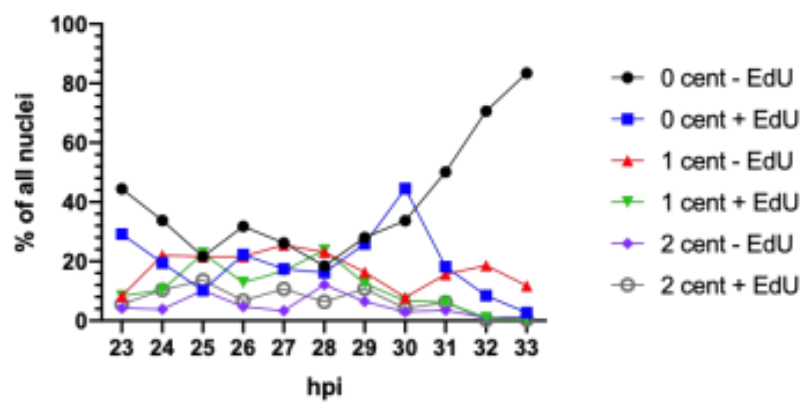


- (which was not certified by peer review) is the author/funder. All rights reserved. No reuse allowed without permission.

Fig 5 A

\section{P.falciparum}

1 nucleus cells

2-6 nuclei cells

$>6$ nuclei cells
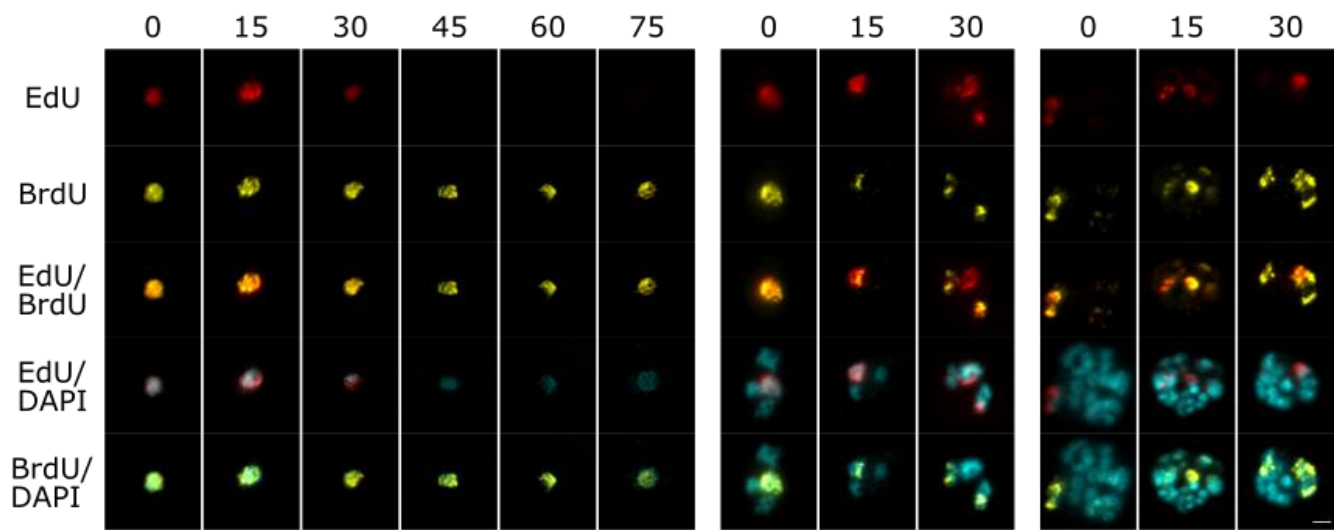

B
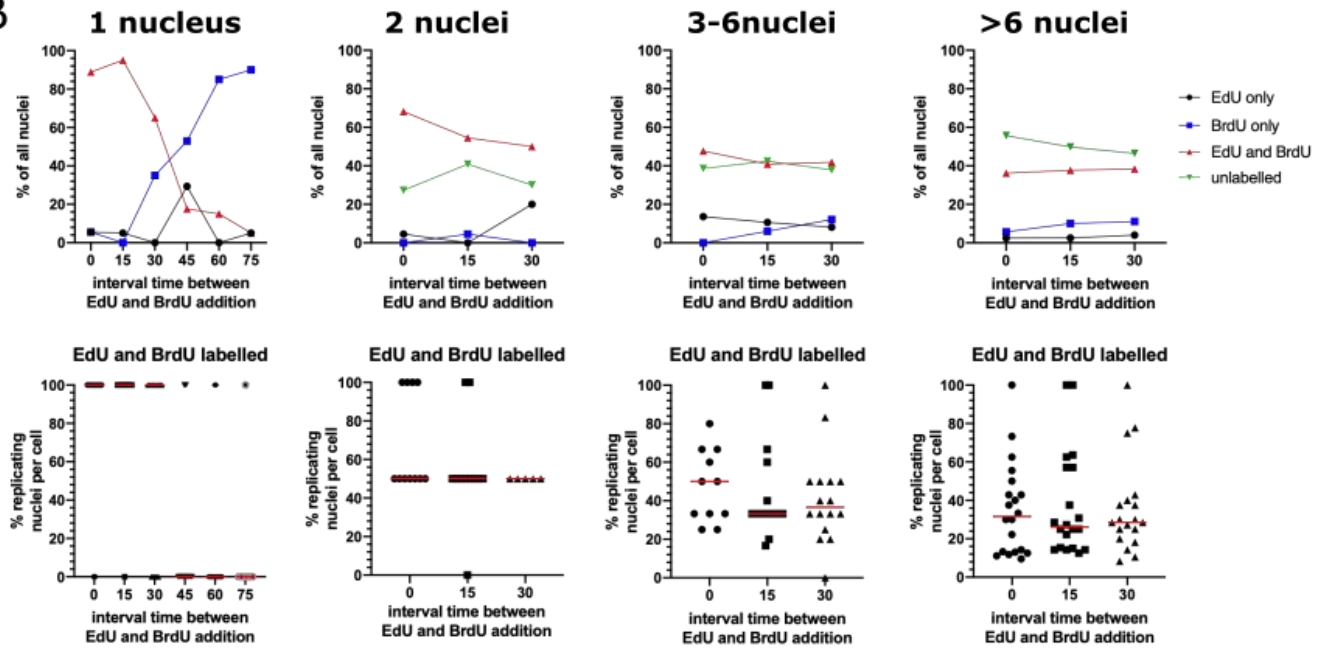

C

\section{P.knowlesi}

1 nucleus cells

2-6 nuclei cells

$>6$ nuclei cells
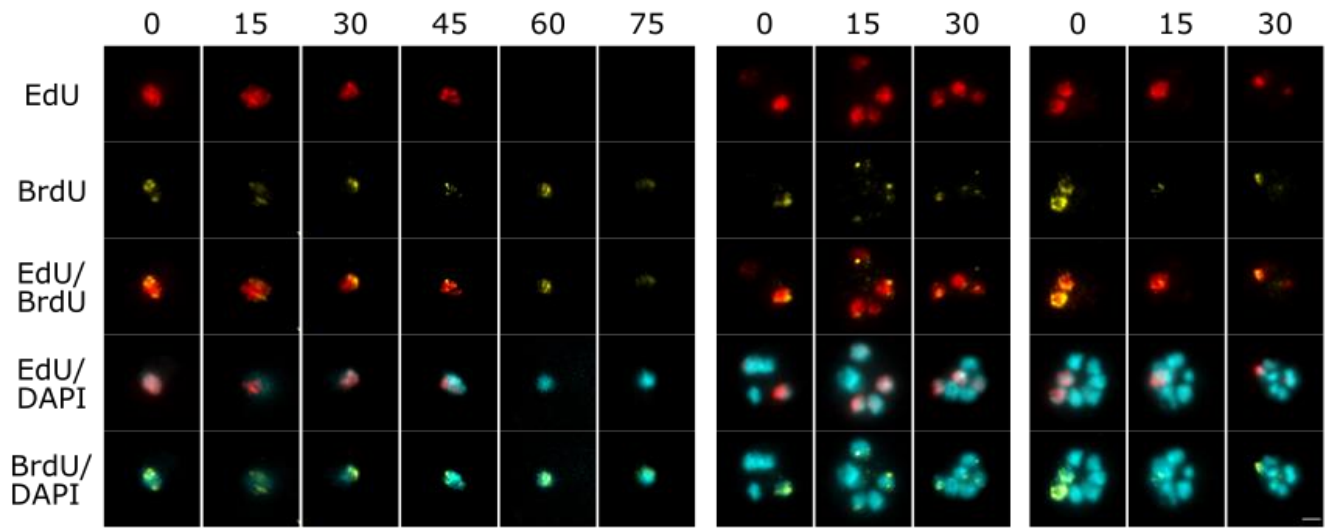

D 1 nucleus

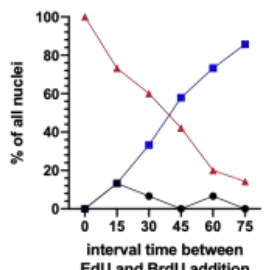

EdU and BrdU labelled

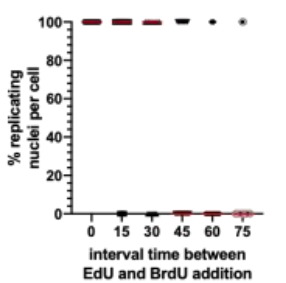

2 nuclei

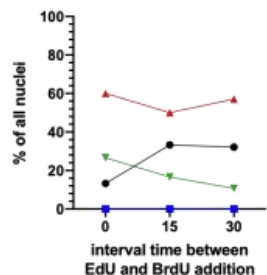

EdU and BrdU labelled

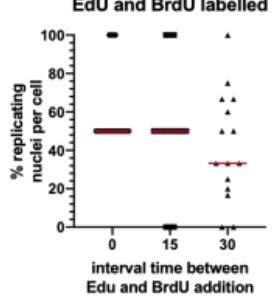

3-6nuclei

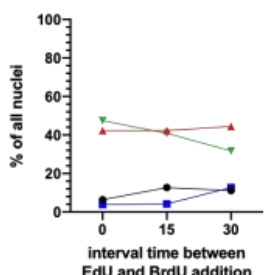

EdU and BrdU labelled

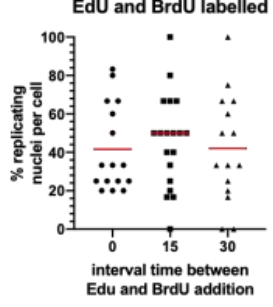

$>6$ nuclei

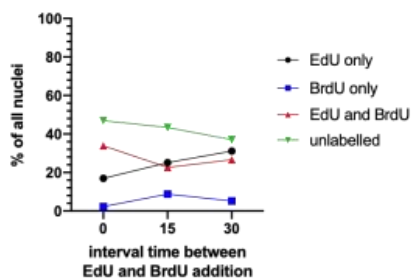

EdU and BrdU labelled

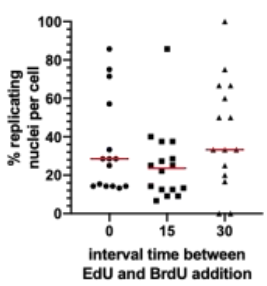




\section{Fig 6}

A

P.falciparum

32-35 35-38 38-41 41-44

$35-4138-44$

$35-44$

EdU
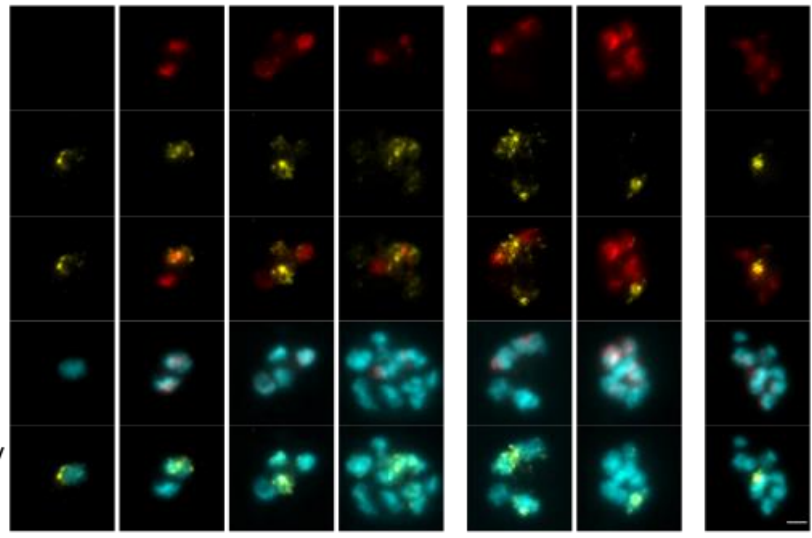

B
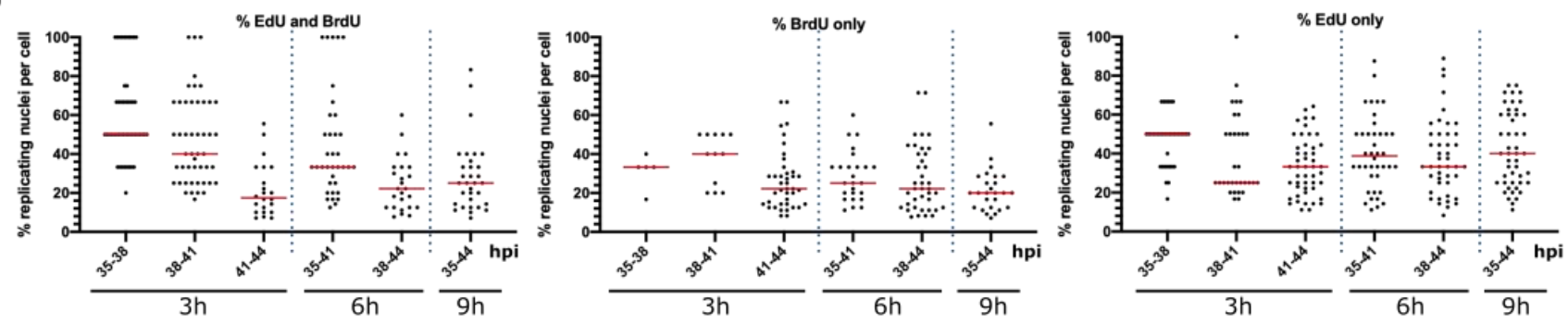

C

\section{P.knowlesi}

$\begin{array}{lllll}18-20 & 20-22 & 22-24 & 24-26 & 26-28\end{array}$

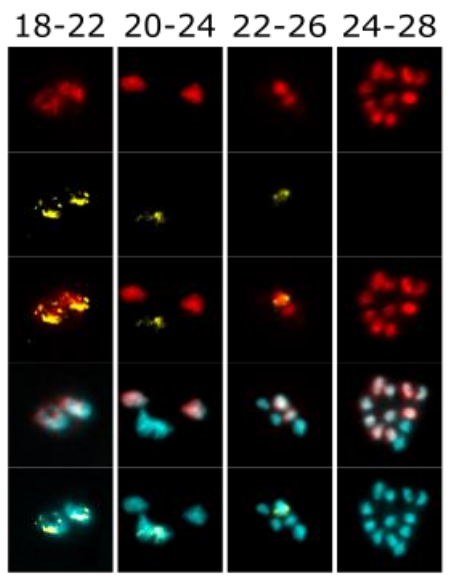

$4 \mathrm{~h}$

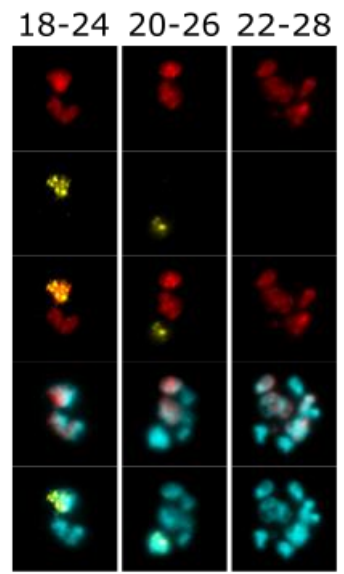

$6 \mathrm{~h}$

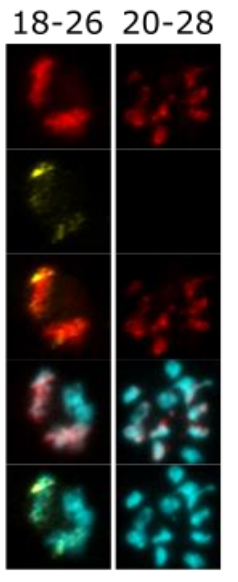

$8 \mathrm{~h}$

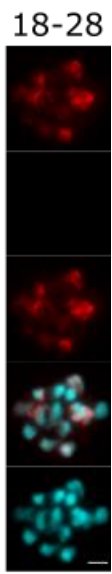

10h

D
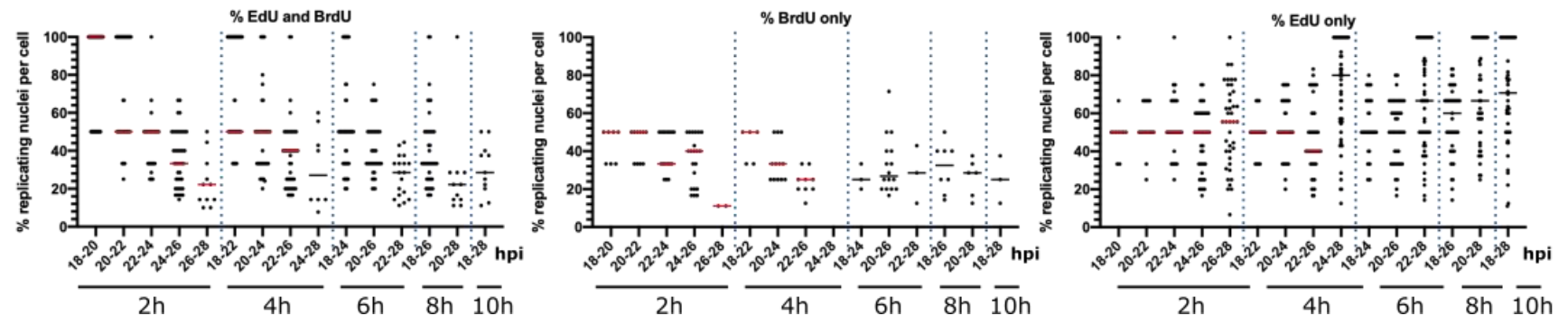


\section{Fig 7}

A

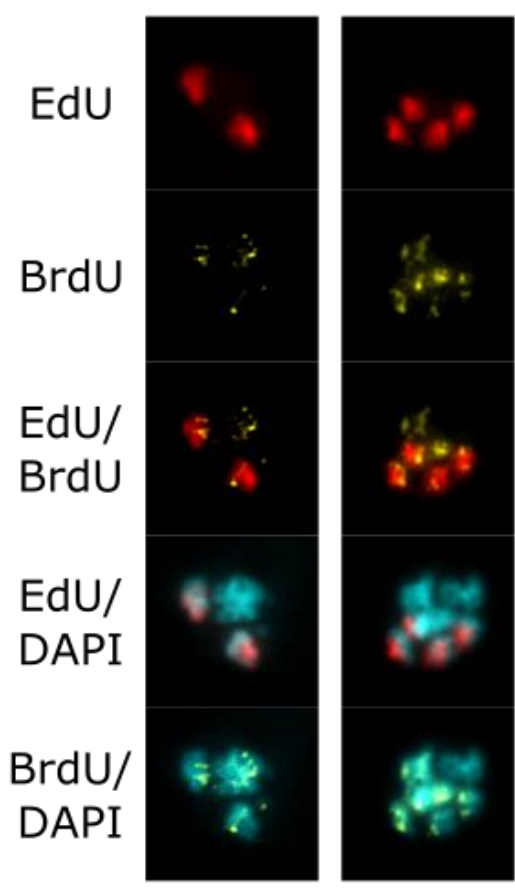

D

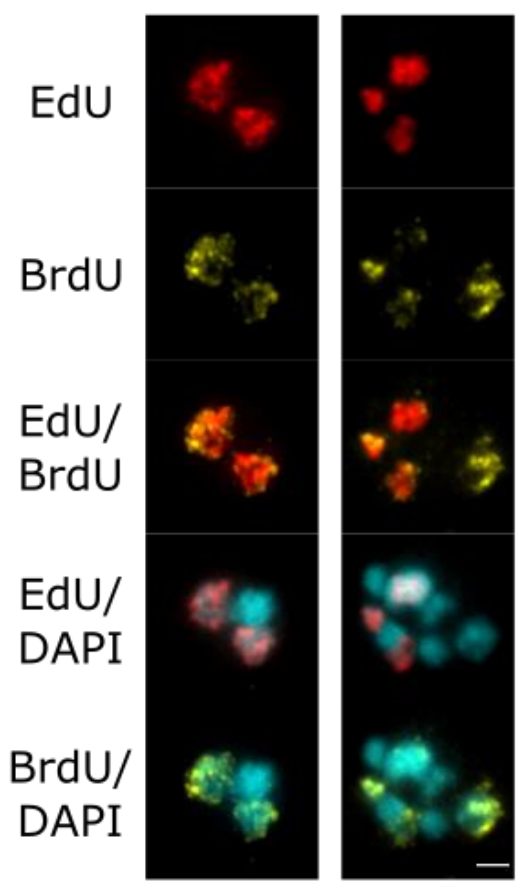

B

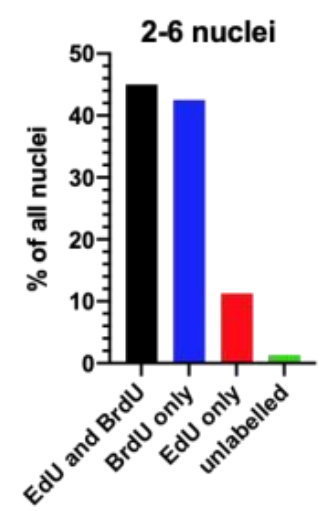

C

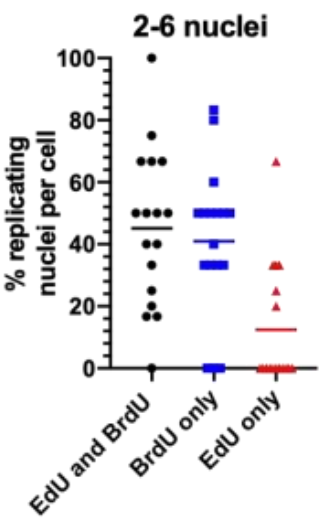

E

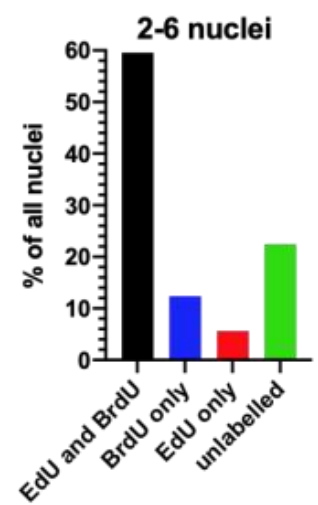

F

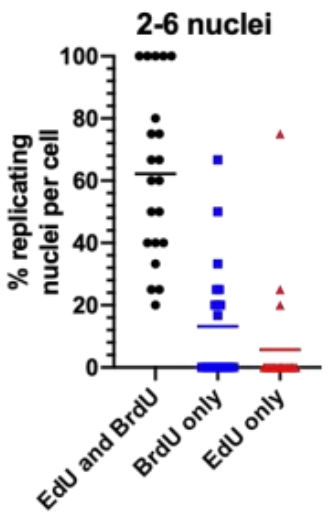

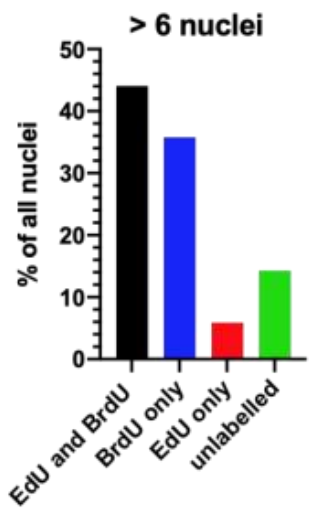
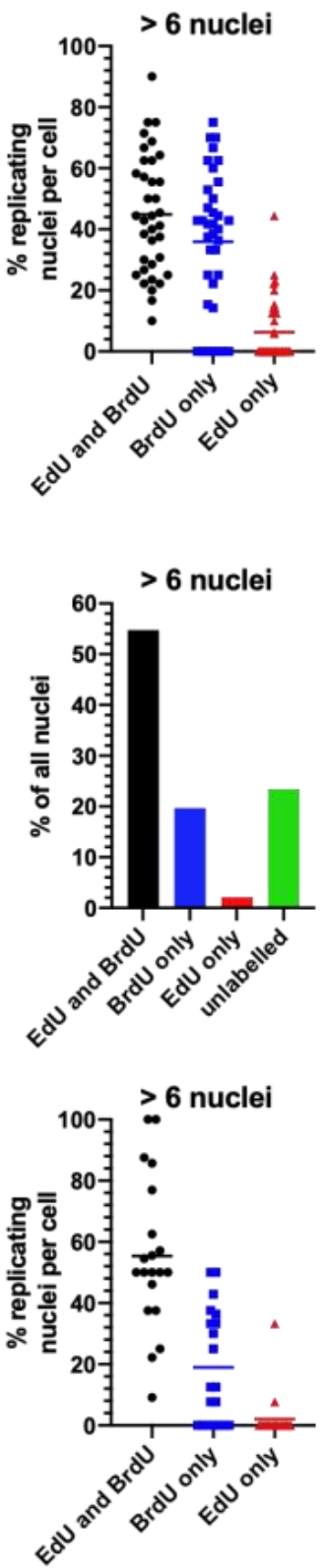
Fig 8

A

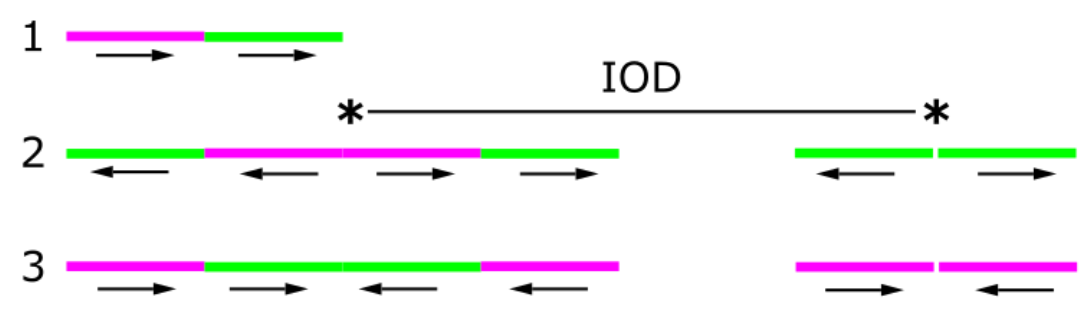

B
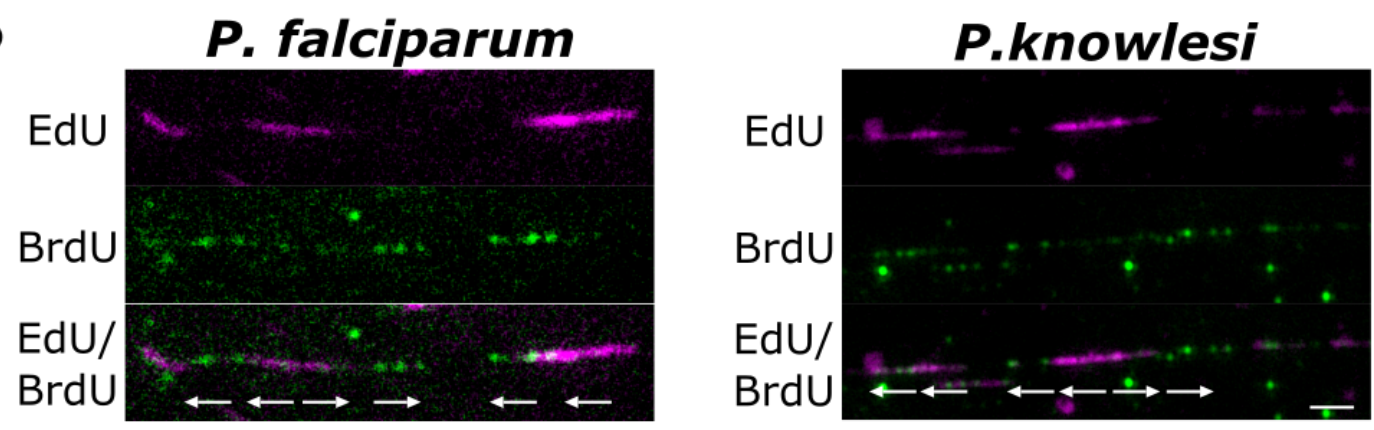

C

Speed

D
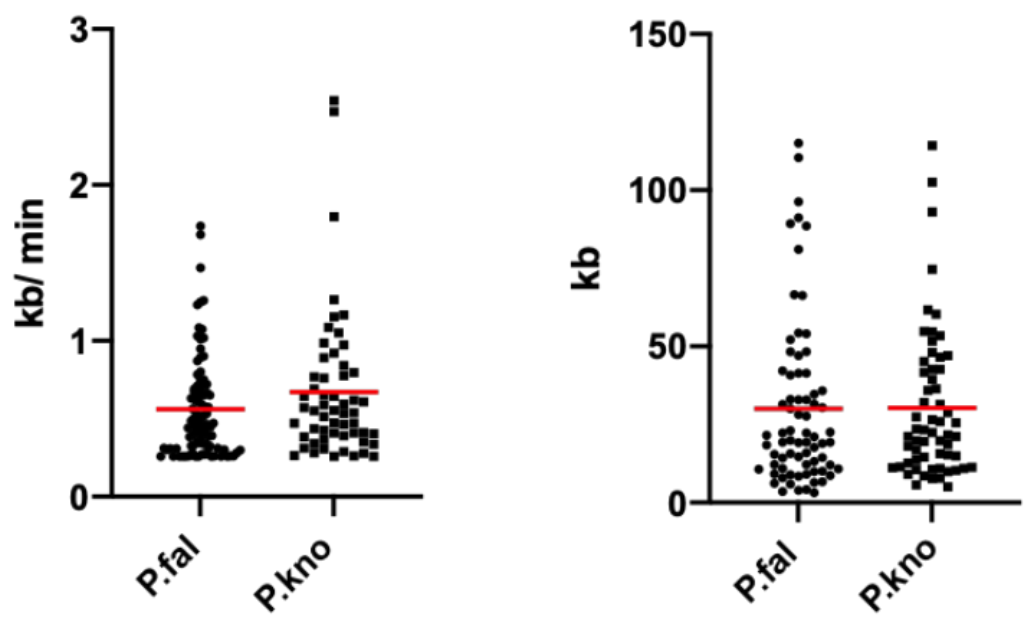
Supp 1

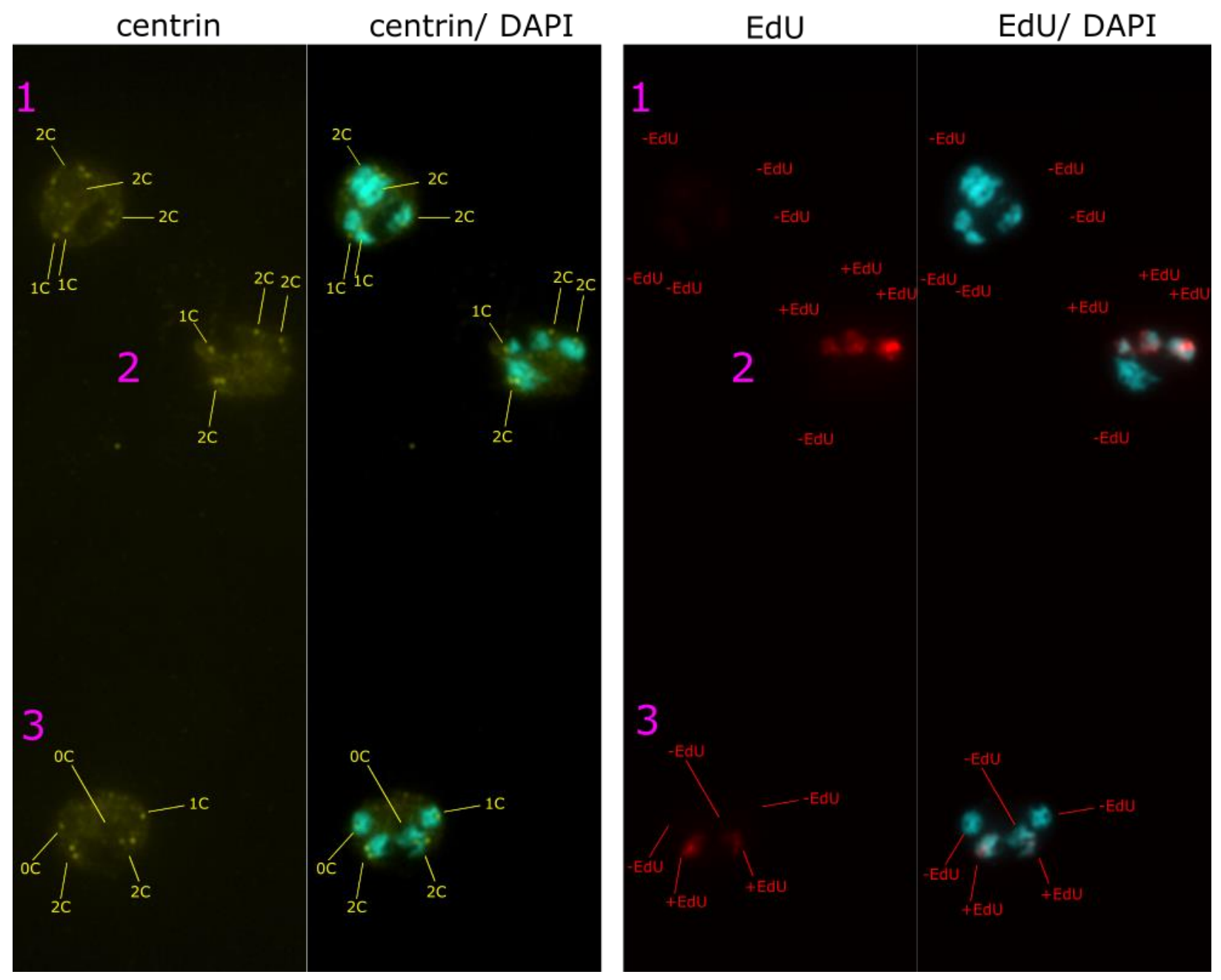

\section{Supplementary figure 1}

Example of a typical slide, showing 3 parasites classified for their number of nuclei, number of centrin foci and presence/absence of EdU staining. 


\section{P. falciparum}

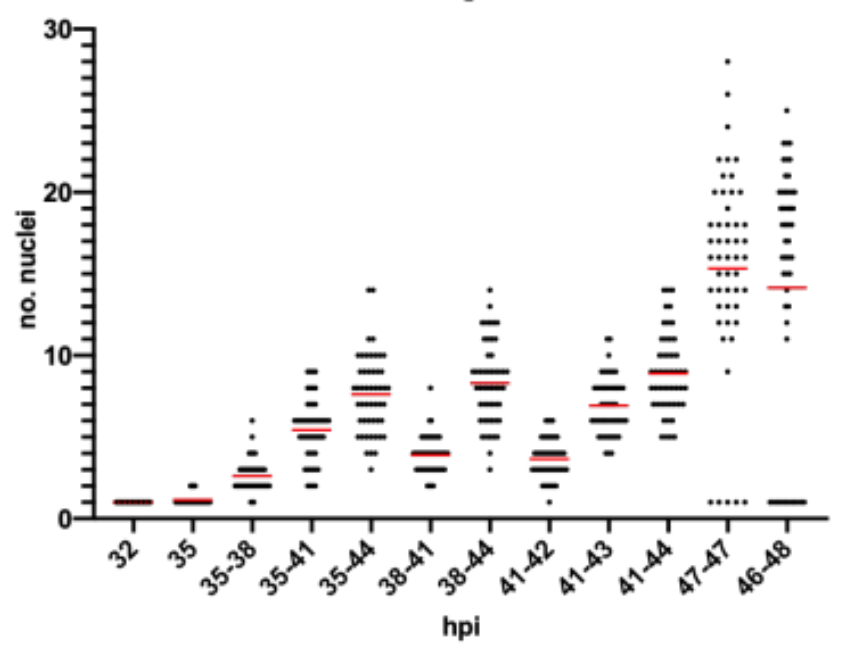

P. knowlesi

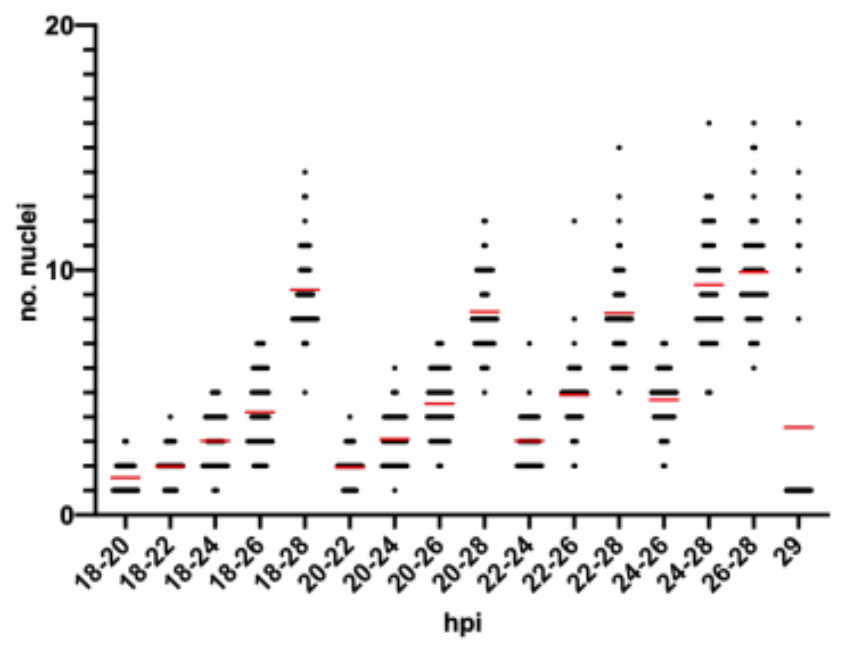

Supplementary figure 2

A: $P$. falciparum scatter graphs of nuclear numbers at each point of the double-labelled timecourse shown in main Figure 6. B: P. knowlesi scatter graphs of nuclear numbers every 2 hours across the double labelled timecourse. Final timepoints show reinvasion. 
A

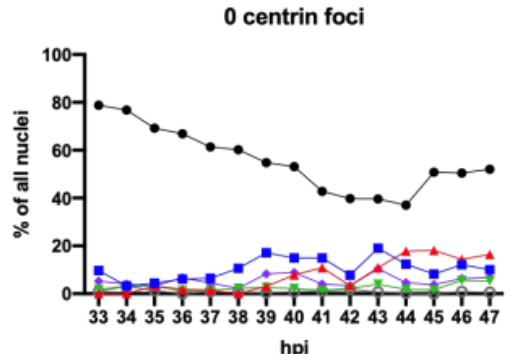

hpi

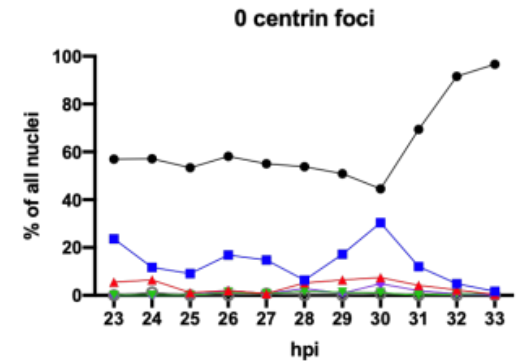

B

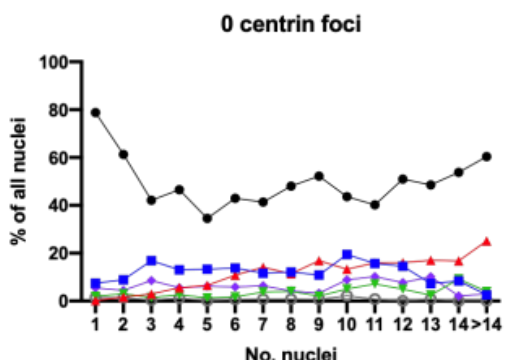

No. nuclei

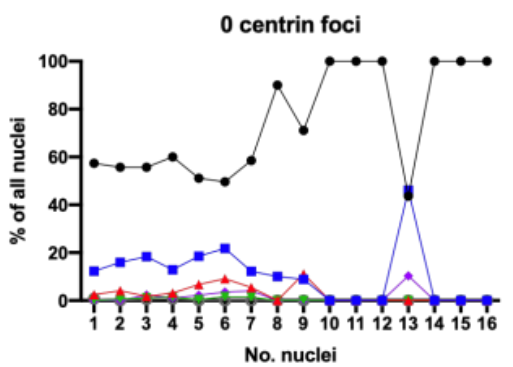

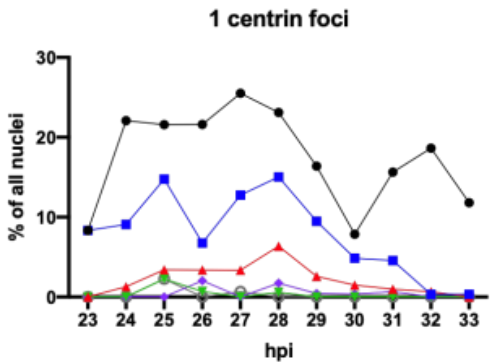

P. falciparum

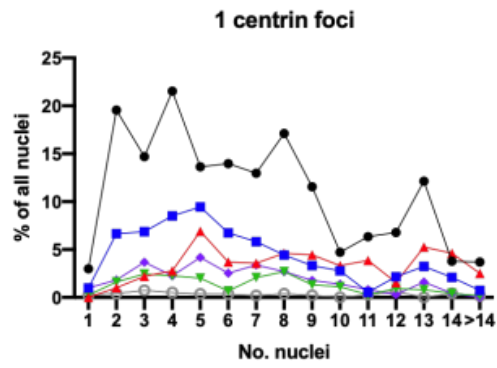

\section{P. knowlesi}

\section{P. falciparum}

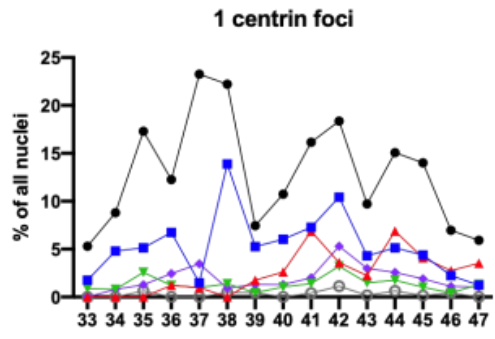

\section{P. knowlesi}

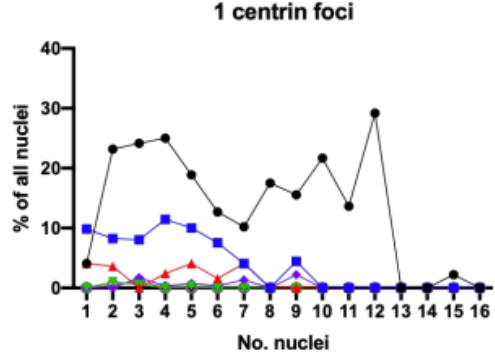

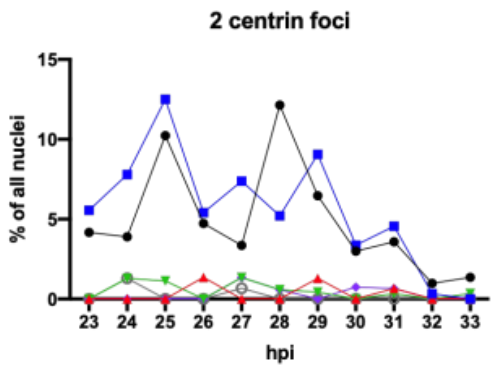
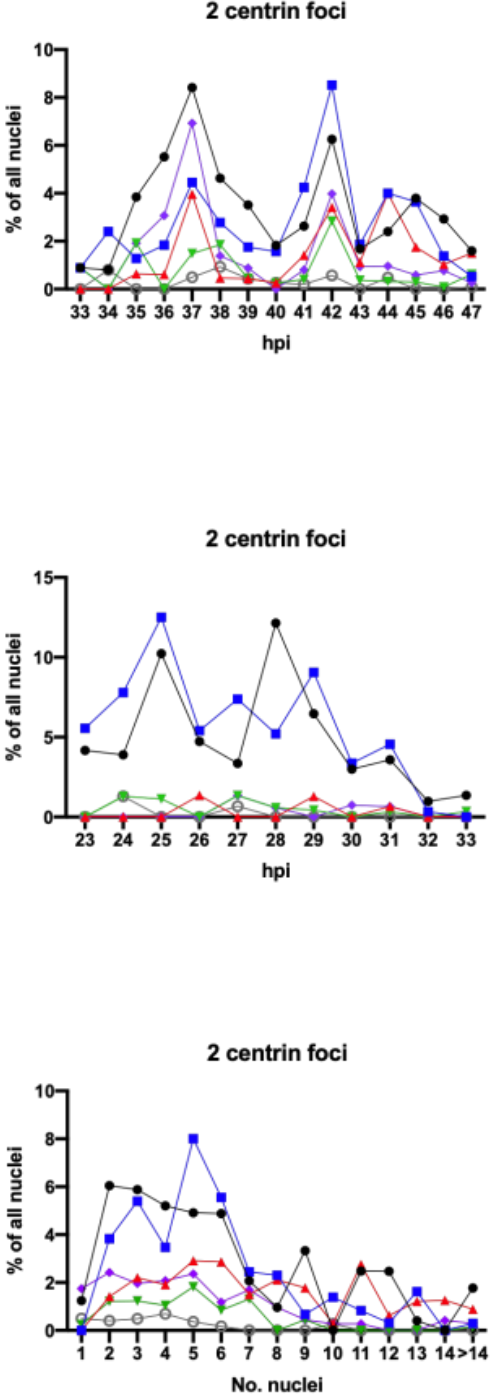

hpi

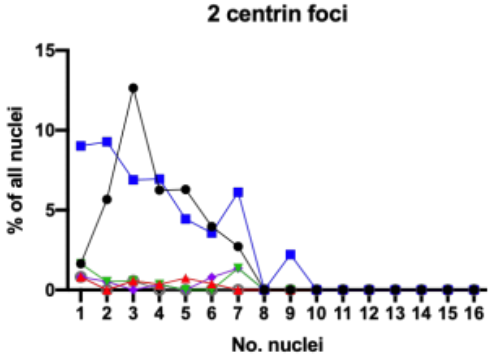

Supplementary figure 3

A: Percentage of nuclei with 0, 1 or 2 centrin foci and patterns of intranuclear DNA replication (full, partial, or discrete foci) throughout schizogony.

B: Data as in A, replotted by number of nuclei per cell rather than hpi.

\footnotetext{
- no stain

- bright fully stained

$\rightarrow$ less bright fully stained

$\rightarrow$ partial<half

$\rightarrow$ partial>half

- - no foci
} 
Supp 4

(which was not certified by peer review) is the author/funder. All rights reserved. No reuse allowed without permission.

P. falciparum

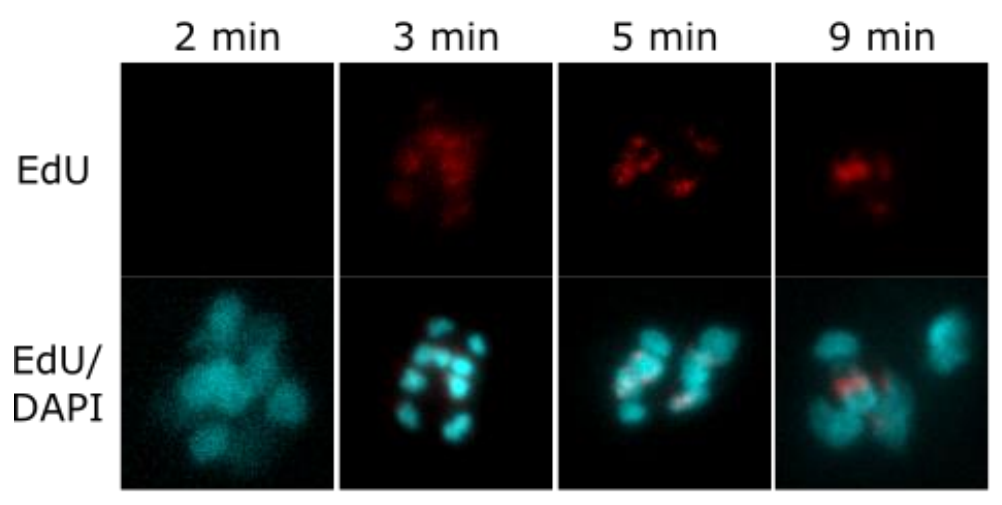

P. knowlesi

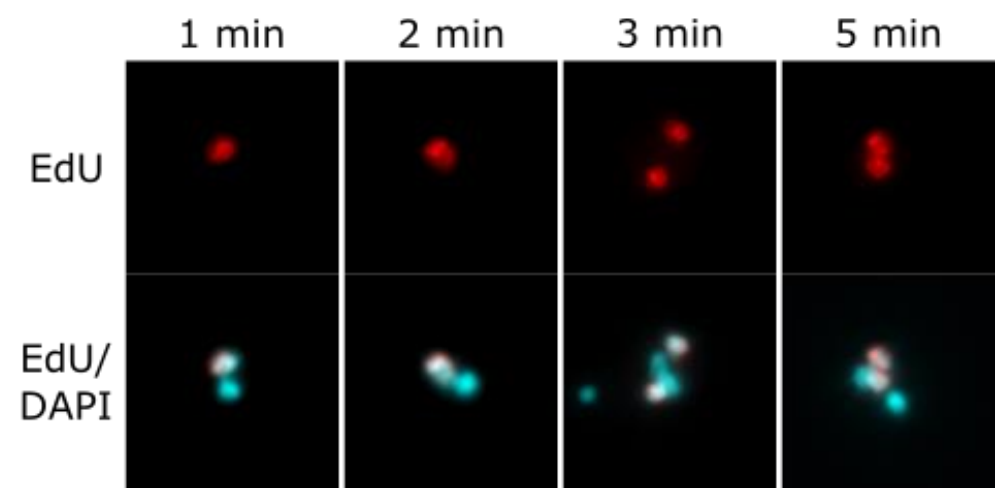

Supplementary figure 4

The minimum pulse-labelling period that can be detected via EdU-labelled DNA was tested. In P. falciparum, 3 minutes was detectable (faintly) and 5 minutes gave reasonably bright signal. In $P$. knowlesi, labelling was clearly detectable within 1 minute. 
Supp 5
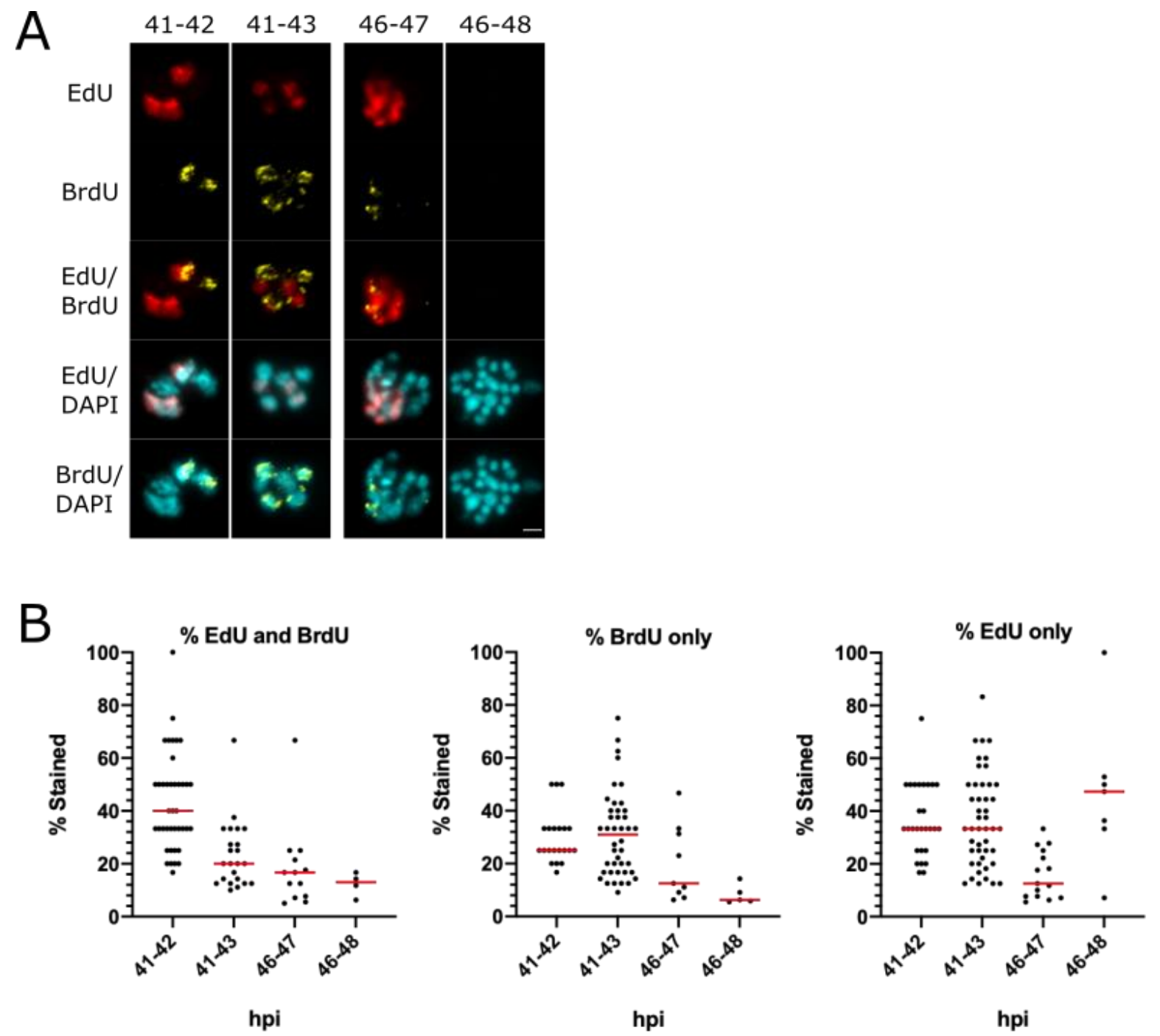

\section{Supplementary figure 5}

A: Representative examples of cells across the double-labelled timecourse with $2 \mathrm{~h}$ intervals for $P$. falciparum. Scale bar $2 \mu \mathrm{m}$. B: Percentages of nuclei labelled with EdU alone, BrdU alone, or both labels throughout the timecourses shown in (A). 
(which was not certified by peer review) is the author/funder. All rights reserved. No reuse allowed without permission.

Supp 6
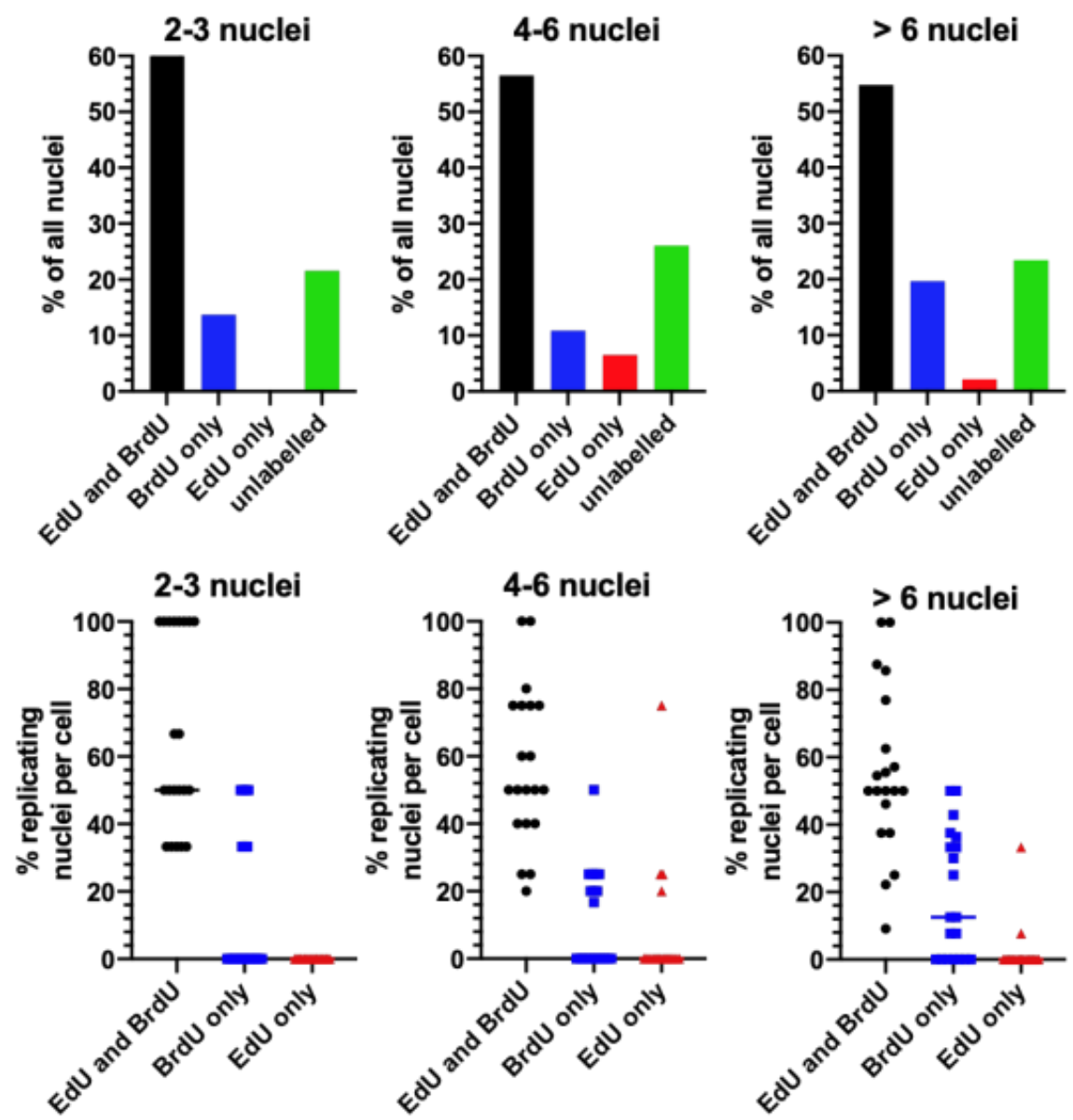

5 hours
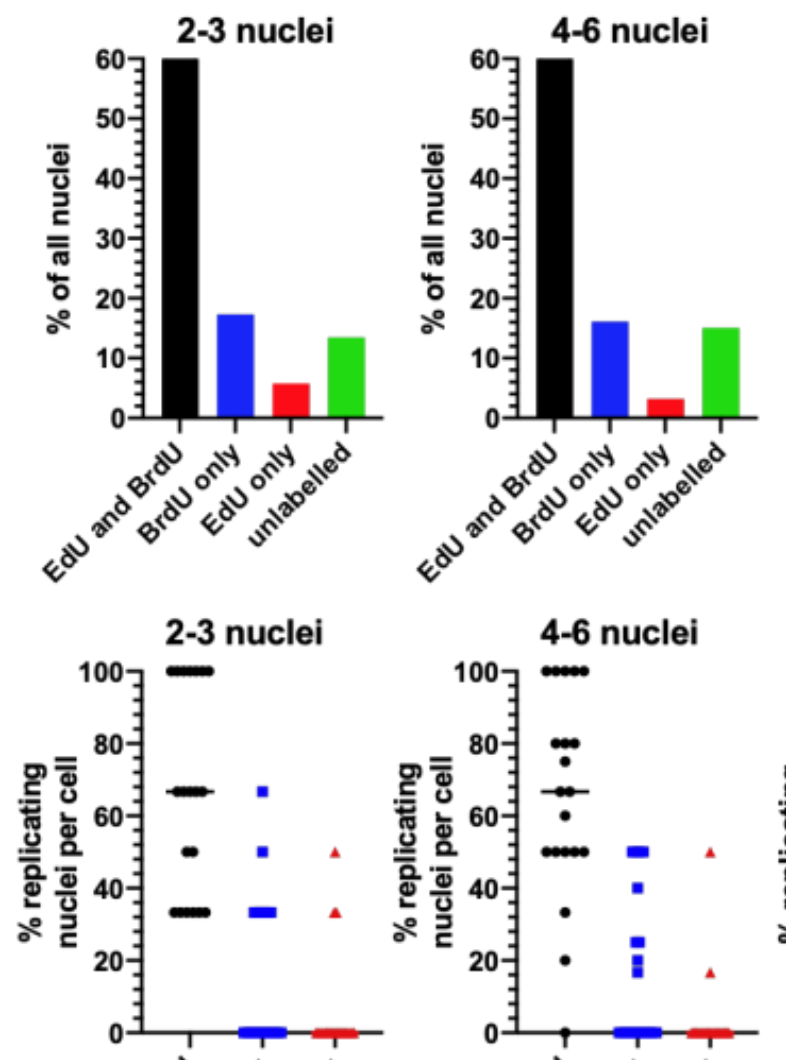
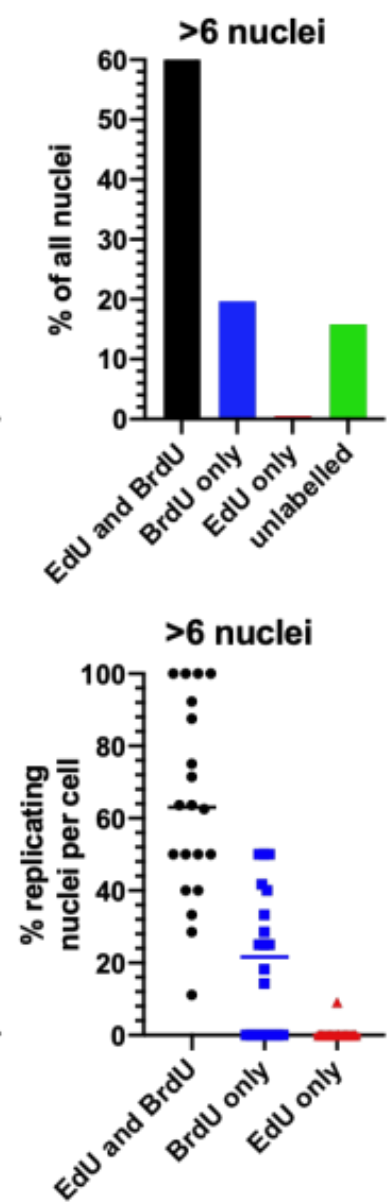

Supplementary figure 6

Data as in main Figure

7 , showing the percentages of $P$.

knowlesi nuclei labelled with EdU and BrdU, EdU alone, BrdU alone, or neither label $(n=20$ cells), after both $2 \mathrm{~h}$ and $5 \mathrm{~h}$ - demonstrating a higher percentage of arrested nuclei after $2 \mathrm{~h}$ ( $22 \%$ of S-phase) than $5 \mathrm{~h}$ ( $>50 \%$ of S-phase), but an overall similar picture.

Data are also stratified into cells with 2-3, 4- 6, or more than 6 nuclei, showing that arrested nuclei are still detected in very young $2-3 n$ schizonts. 